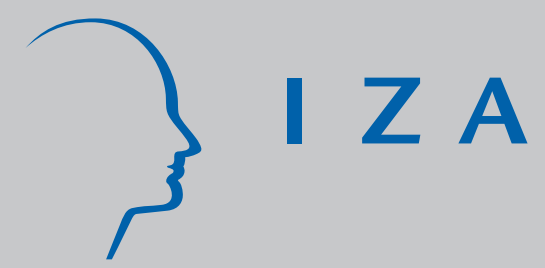

IZA DP No. 7266

Monitoring Job Search Effort with Hyperbolic Time Preferences and Non-Compliance: A Welfare Analysis

Bart Cockx

Corinna Ghirelli

Bruno Van der Linden

March 2013 


\title{
Monitoring Job Search Effort with Hyperbolic Time Preferences and Non-Compliance: A Welfare Analysis
}

\author{
Bart Cockx \\ SHERPPA, Ghent University, \\ IRES, IZA and CESifo \\ Corinna Ghirelli \\ SHERPPA, Ghent University
}

\author{
Bruno Van der Linden \\ IRES, Université Catholique de Louvain, \\ FNRS, IZA and CESifo
}

\author{
Discussion Paper No. 7266 \\ March 2013
}

\author{
IZA \\ P.O. Box 7240 \\ 53072 Bonn \\ Germany \\ Phone: +49-228-3894-0 \\ Fax: +49-228-3894-180 \\ E-mail: iza@iza.org
}

\begin{abstract}
Any opinions expressed here are those of the author(s) and not those of IZA. Research published in this series may include views on policy, but the institute itself takes no institutional policy positions. The IZA research network is committed to the IZA Guiding Principles of Research Integrity.

The Institute for the Study of Labor (IZA) in Bonn is a local and virtual international research center and a place of communication between science, politics and business. IZA is an independent nonprofit organization supported by Deutsche Post Foundation. The center is associated with the University of Bonn and offers a stimulating research environment through its international network, workshops and conferences, data service, project support, research visits and doctoral program. IZA engages in (i) original and internationally competitive research in all fields of labor economics, (ii) development of policy concepts, and (iii) dissemination of research results and concepts to the interested public.
\end{abstract}

IZA Discussion Papers often represent preliminary work and are circulated to encourage discussion. Citation of such a paper should account for its provisional character. A revised version may be available directly from the author. 


\section{ABSTRACT \\ Monitoring Job Search Effort with Hyperbolic Time Preferences and Non-Compliance: A Welfare Analysis}

This paper develops a partial equilibrium job search model to study the behavioral and welfare implications of an Unemployment Insurance (UI) scheme in which job search requirements are imposed on UI recipients with hyperbolic preferences. We show that, if the search requirements are well chosen, a perfect monitoring scheme can in principle increase the job finding rate and, contrary to what happens with exponential discounting, it can raise the expected lifetime utility of the current and future selves of sophisticated hyperbolic discounters. The same holds for naive agents if the welfare criterion ignores their misperception problem. In sum, introducing a perfect monitoring scheme can be a Pareto improvement. However, if claimants have the opportunity to withdraw from the UI scheme, their long-run utility can even be lower than in the absence of job search requirements. Imperfections in the measurement of job-search effort further reduce the chances that monitoring raises the welfare of the unemployed.

JEL Classification: $\quad$ D60, D90, J64, J65, J68

Keywords: job search model, job search monitoring, non-compliance, hyperbolic discounting, social efficiency

Corresponding author:

Bart Cockx

SHERPPA

Ghent University

Tweekerkenstraat 2

9000 Gent

Belgium

E-mail: Bart.Cockx@UGent.be 


\section{Introduction}

Long-term unemployment is a major problem, in particular in a number of European labor markets (OECD, 2011). This pattern comes along with the evidence of a very low search activity exerted by the unemployed (Manning, 2011, Krueger and Mueller, 2010). It is well known that the provision of Unemployment Insurance (UI) raises moral hazard problems, i.e. the more generous UI, the lower the search incentives for the unemployed (e.g. Lalive et al., 2006). Many countries impose job search requirements on benefit recipients to cope with moral hazard in UI (OECD, 2007). To verify compliance, job search effort is monitored and, in case of non-compliance, benefit recipients are sanctioned. However, as any policy addressing moral hazard, monitoring involves an insuranceefficiency trade-off (Boone and van Ours, 2006, Boone et al., 2007; Cockx et al., 2011). Restoring incentives comes at a cost of reducing the capacity of UI to adequately insure workers against the risk of unemployment. Different from other policy instruments, monitoring of job search does not directly affect the unemployment benefit (UB) level. However, monitoring increases job search costs and decreases the average quality of prospective jobs, since rational forward looking unemployed workers typically reduce their reservation wage in response to the higher job search requirements. Hence, the expected lifetime utility of the unemployed is negatively affected.

These results apply for individuals with standard exponential time preferences. These individuals discount the future at a constant rate and, hence, behave consistently over time. However, both laboratory experiments and empirical studies find evidence that procrastination in inter-temporal choices is common (e.g. see Ainslie, 1992; Loewenstein and Thaler, 1989; Thaler and Shefrin, 1981 ; for a critical review see Frederick et al., 2002). That is, people seem to show self-control problems whenever they have to commit to a plan entailing present costs and future rewards (or vice versa). They may keep postponing the costly task over time and end up not achieving the future rewards, even if it was rationally optimal to reach it. This is evidence of hyperbolic discounting. Individuals exhibit a high degree of discounting in the short-run and a relatively low degree of discounting in the long-run. To cope with this limitation, a new branch of economics has been investigating inter-temporal choices under the assumption of hyperbolic time preferences (e.g., Loewenstein and Prelec, 1992, O’Donoghue and Rabin, 1999) 1

Recently, based on a longitudinal experiment on inter-temporal effort choice, Augenblick et al. (2013) find limited evidence of present bias in choices over monetary payments. By contrast, individuals procrastinate substantially in effort choices. Moreover, these individuals are more likely to

\footnotetext{
${ }^{1}$ Researchers have studied the implications of this different behavioral assumption on various economic decisions. For instance, among others Laibson (1997) and Angeletos et al. (2001) examined saving-consumption decisions, while Carrillo and Mariotti (2000) focused on learning decisions and Fang and Silverman (2009) on labor supply and welfare participation. Others investigated specific consumption decisions: e.g. Mullainathan and Gruber (2005) focused on smoking, Fang and Wang (2010) on preventive health care, while DellaVigna and Malmendier (2006) studied contract choices and attendance to health clubs.
} 
choose for a commitment device that forces them to complete more effort than they instantaneously desire, since they are aware of their present bias and take actions to limit their future behavior, i.e. they are sophisticated hyperbolic agents. This is consistent with earlier research of DellaVigna and Paserman (2005) - hereafter referred as PDV - and Paserman (2008) who find evidence that hyperbolic preferences are particularly relevant in explaining the observed patterns of job search behavior in the US ${ }^{2}$ Job search effort typically entails immediate costs and delayed benefits. Consequently, individuals with hyperbolic preferences are always tempted to delay job search. Since unemployed workers engage in too little job search, PVD show ${ }^{3}$ that they are willing to pay a positive price for a commitment device that forces them to search more intensively if they are sophisticated hyperbolic agents. Job search monitoring could be such a commitment device. Based on simulations of an estimated structural job search model on US data, Paserman (2008) has indeed demonstrated that, if workers are impatient, monitoring job search can improve their long-run utility by lowering expected unemployment duration and raising expected wages. In other words, to the extent that monitoring is relatively cheap to implement 4 it can unambiguously lower government expenditures and increase social welfare without facing an insurance-efficiency trade-off. This contrasts with the conclusions for unemployed people with exponential time preferences.

Empirical evidence does not unambiguously support these positive conclusions with regards to monitoring of job search: neither the job finding rate nor the job quality always increase, and sometimes the unemployed rather exit to inactivity. For instance, Klepinger (1998), McVicar (2008) and Cockx and Dejemeppe (2012) find that monitoring enhances the job finding rate. By contrast, Ashenfelter et al. (2005) find that tighter search requirements have insignificant effects on transitions to employment and Klepinger et al. (2002) even find negative effects. In addition, Petrongolo (2009) reports negative impacts on the job quality (mainly earnings and employment duration) and, together with Manning (2009), she reports evidence that it leads to flows out of the UB claimant status.

In this paper we show that these ambiguous findings on the effectiveness of job search monitoring need not be incompatible if the unemployed behave as agents with hyperbolic time preferences. This is because the decision to comply with the imposed job requirements does not depend on the longrun utility of these agents, but rather on the short-run utility of the current self for whom the benefits of enhanced search are shown to be smaller 5 than for the future self. Consequently, even if job search requirements are set at a sub-optimal, i.e. too low, level from the perspective of the future self, unemployed hyperbolic discounters may still stop complying if search requirements become too demanding. Hence, it is shown that increasing job search requirements to a level that is optimal from the perspective of the future selves or from the perspective of society may after all still lead

\footnotetext{
2 Halima and Halima (2009) find similar evidence for France.

${ }^{3}$ See their Proposition 1.

${ }^{4}$ See Boone et al. (2007) and Cockx et al. (2011) for some estimations of these costs.

${ }^{5}$ Even nonexistent for a naive hyperbolic agent, as will be shown below.
} 
to a sub-optimal level of search effort and a long-run utility that is even lower than in the absence of job search requirements. Furthermore, we show that imperfections in the monitoring technology induced by measurement error reinforce this problem.

The policy implication of this analysis is that job search monitoring may improve social welfare unambiguously only if the job search requirements are not set at a too high level. Moreover, it is shown that, as a consequence, job search monitoring may not be socially efficient, implying that other policy instruments, such as counseling, may perform better from a social welfare point of view.

The model is based on an extension of the basic partial equilibrium job search model (Mortensen, 1986) in three directions. First, we introduce hyperbolic discounting as in PDV. We consider both agents with naive and sophisticated hyperbolic preferences. Second, we include in this model a perfect job search monitoring scheme in a very similar way as Manning (2009) and Petrongolo (2009) do for individuals with exponential preferences. Finally, we allow for imperfections in the monitoring technology by allowing for measurement error (see e.g. Boone et al., 2007, Cockx et al., 2011). Within this model we show that the aforementioned results of PDV and Paserman (2008) regarding the social efficiency of monitoring do not only apply for risk neutral, but also for risk averse worker: ${ }^{6}$ and apply for a more general class of wage offer distributions than the ones they considered. We also contribute to the literature on hyperbolic discounting by developing a graphical exposition of the impact of hyperbolic preferences on the choice of job search effort and the reservation wage. In addition, this graphical exposition contributes to a better intuitive understanding of the main results of this paper.

The rest of the paper is organized as follows. The notion of hyperbolic preferences is succinctly introduced in Section 2, We explain the difference between a sophisticated and a naive agent. Section 3 describes the benchmark model. In this model the monitoring technology is assumed to be perfect. We describe the assumptions and notations, the optimization problem of both the naive and the sophisticated agent and present the first order conditions of the solution. We devote a separate section to the graphical analysis of the solution, since we believe that this enhances the intuition of our findings. In Section 5 we demonstrate why raising job search effort of unemployed individuals with hyperbolic preferences can be socially efficient and we discuss how non-compliance affects this property. In Section 6 we generalize the model by incorporating an imperfect monitoring technology. All propositions are proved in the Appendix.

\footnotetext{
${ }^{6}$ The experimental literature that provides evidence of hyperbolic discounting has been criticized for the reason that this behavior is compatible with exponential discounting provided that the marginal utility of consumption is decreasing (see e.g. Noor 2009). In our paper we assume that agents are impatient even if the marginal utility of consumption is decreasing.
} 


\section{Hyperbolic preferences}

The literature on hyperbolic discounting starts with Strotz (1955), Pollak (1968) and Goldman (1980). Hyperbolic discounting is used to model individuals with present-biased preferences, who are more impatient in the short run and less impatient in the long run. Agents with such preferences outweigh the immediate costs compared to the distant rewards and thus postpone costly activities to the future. Present-biased preferences can be represented by the following hyperbolic discount function introduced by Phelps and Pollak (1968):

$$
V(c)=u\left(c_{0}\right)+\beta \delta u\left(c_{1}\right)+\beta \delta^{2} u\left(c_{2}\right)+\ldots+\beta \delta^{t} u\left(c_{t}\right) \ldots+\beta \delta^{T} u\left(c_{T}\right)=u\left(c_{0}\right)+\sum_{t=1}^{T} \beta \delta^{t} u\left(c_{t}\right)
$$

where $V(c)$ is the utility function over consumption streams $c=\left(c_{0}, \ldots, c_{t}, \ldots c_{T}\right)$ for $t \in\{0,1,2, \ldots, T\}$ and where $\beta \leq 1$ and $\delta<1$ are the short-term and long-term discount parameter, respectively. From the perspective of $t=0$, a trade-off between $t=0$ and $t=1$ is discounted at the short-term discount factor $\beta \delta$, whereas a trade-off between any two future periods is discounted at the the long-term discount factor $\delta$. Under hyperbolic discounting, $\beta<1$ and the agents are more impatient in evaluating short-term trade-offs than long-term ones $(\delta>\beta \delta)$. In contrast, $\beta=1$ corresponds to the traditional case of exponential discounting, where all periods are discounted in the same way.

According to the literature on hyperbolic discounting, the dynamic optimization problem of an agent is typically represented as an interpersonal game, the players being all the different selves of the agent at different points in time. All future selves are identical and discount the future according to parameter $\delta$. The current self instead differs from the latter because he discounts the future by $\beta \delta<\delta$. Each self wants to delegate search to the other selves - in particular the current self wants to postpone search to the future - and the solution is specified as an equilibrium of the optimal strategies in this game.

The literature distinguishes two types of hyperbolic agents, a sophisticated and a naive one. They differ in the perception of how their respective future selves will behave. A sophisticated agent correctly realizes that his future selves will act exactly as the current self (discounting by $\beta \delta$ ), while a naive agent wrongly believes that his future selves will behave as an exponential agent (discounting by $\delta$ ). Using the terminology of (Gruber and Köszegi, 2000, 2001) they both have a self-control problem, but only the naive agent has a misperception problem. Two arguments are in favor of the assumption of sophistication in hyperbolic discounting. First, sophistication implies that the agents have rational expectations about their future behavior, which is a natural assumption in economics (O'Donoghue and Rabin, 1999). Second, the broad use of commitment devices (e.g. see Schelling, 1984) suggests that individuals are aware of their self-control problems, and this is evidence of a certain degree of sophistication. In recent experimental research, Augenblick et al. (2013) finds further support for the preference for commitment relative to flexibility and therefore for sophistication. For purposes of completeness, we will nevertheless consider the behavior of both 
types of agents in the analysis below.

\section{The Benchmark Model}

\subsection{Assumptions and Notations}

We develop a partial equilibrium job search model under hyperbolic preferences in a stationary discrete time setting..$^{7}$ Infinitely-lived unemployed workers choose their reservation wage $x$ and a scalar search-effort intensity $\sigma$ to maximize their expected discounted lifetime utility. Unemployed workers are entitled to a flat rate unemployment benefit (UB). The total income while unemployed $b>0$ is equal to the UB plus any other external income (e.g. income from a partner). The payment of UB is conditional on a search requirement $\bar{\sigma}>0$. The job search effort of the UB claimant is monitored at the end of each period, after job arrival and acceptance decision. Hence, job search effort is monitored if no offer has been received or if an offer has been rejected 8 If the benefit claimant does not comply with this requirement, UB is withdrawn permanently as from the subsequent period. The exogenous income of sanctioned individuals is the external income plus, depending on the institutional context, income provided by relatives, by charities and/or a (meanstested) assistance benefit. The income of sanctioned individuals is denoted $z<b$. We assume that job search of sanctioned individuals is no longer monitored and is therefore chosen freely. In our stylized benchmark representation, we assume that monitoring is perfect, meaning that job search effort is observed with perfect precision and that, if search effort falls below the requirement $\bar{\sigma}$, a sanction is imposed with probability one. This stylized representation of a monitoring scheme has also been adopted by other researchers (Manning, 2009, Petrongolo, 2009, e.g.). In section 6 we study the consequences of some imperfection in the monitoring technology.

While PVD and Paserman (2008) consider risk-neutral individuals, we assume, more realistically, that they are risk averse. As is standard in the job search literature, we assume that the instantaneous utility is separable in income and search effort. Consequently, since we assume that all income is immediately consumed, $u(b)-c(\sigma)$ is the instantaneous net utility of the unemployed, where $u(b)$ denotes the instantaneous utility of consumption and $c(\sigma) \geq 0$ denotes the disutility of search. We make the standard assumptions that $u^{\prime}(\cdot)>0, u^{\prime \prime}(\cdot) \leq 0, c(0)=0, c^{\prime}(\sigma)>0$ and $c^{\prime \prime}(\sigma)>0$.

In each period job offers arrive with a probability that increases with job search effort: $\lambda(\sigma)>0$, with $\lambda(0)=0, \lambda^{\prime}(\sigma)>0$ and $\lambda^{\prime \prime}(\sigma) \leq 0$. The net wage associated to a job offer is randomly drawn

\footnotetext{
${ }^{7}$ Appendix $D$ of PDV demonstrated that the optimization problem of the unemployed worker is equivalent under discrete and continuous setting. We opt for a discrete timing, both for simplicity and to be in accordance with PDV, which is our starting point.

${ }^{8}$ As most job offers are frequently not transmitted by the Public Employment Service, it is assumed that acceptance and rejection of offers are not monitored. Engström et al. (2012) analyze such monitoring.
} 
from an exogenous cumulative wage offer distribution $F(w)$ defined on $[\underline{w}, \bar{w}] \in \mathbb{R}^{+}$. The disutility of effort in employment is normalized to zero, so that the instantaneous net utility in employment is given by $u(w)$, where $w$ denotes the net wage. Employed individuals are laid-off with an exogenous probability $q \in(0,1)$.

\subsection{The Optimization Problem}

We consider the optimization problem of an unemployed worker. In order to capture hyperbolic preferences, we need to distinguish between the lifetime utility of the unemployed current self (referred to by superscript $c$ ) and the lifetime utility of the unemployed and employed future selves viewed from the point of the current self (referred to by superscript $f$ ). We first consider the optimization problem of the current self as a function of the continuation payoffs of the future selves as viewed from the perspective of the current self. Subsequently, we state the optimization problems of the future selves. Since all parameters of the model are time-invariant, we can treat this (dynamic) model as stationary, i.e. avoiding a time subscript 9

An unemployed worker maximizes expected lifetime utility with respect to three choices in the following order: (i) the decision to comply or not to the search requirement $\bar{\sigma}$; (ii) the job search intensity $\sigma$ to set; (iii) to accept or not, if a job is offered. These choices involve very different intertemporal trade-offs. If the job search requirement is binding, the decision to comply induces an instantaneous increase in search costs that should be balanced with the expected future benefit stream to which one remains entitled by not being sanctioned as from the subsequent time period. The choice regarding the level of the job search intensity involves a comparison of job search costs that materialize immediately and benefits that are realized in the future, once a job is found. Accepting a job or continuing search does not impose immediate costs or generate immediate benefits, but affects the stream of benefits and costs that will realize during the future working life. This means that the first and the second choice involve a comparison of short- and long-term pay-offs, whereas the last consists in trading-off long-term utility streams only. This means that short-term impatience $\beta$ matters for the first two decisions, but not for the last. This difference will have important implications for the optimal behaviour of impatient agents.

The aforementioned choice problem can be formalized by the following optimization problem. For $j \in\{s, n, e\}$ :

$$
\begin{aligned}
\tilde{\Omega}_{j}^{c} & =\max _{j}\left\{B_{j}^{c}(\bar{\sigma}), \tilde{Z}_{j}^{c}\right\} \\
B_{j}^{c}(\bar{\sigma}) & =\max _{\sigma \geq \bar{\sigma}} W\left(\sigma, B_{j}^{f}(\bar{\sigma}) \mid b, \beta \delta\right) \\
\tilde{Z}_{j}^{c} & =\max _{\sigma} W\left(\sigma, Z_{j}^{f} \mid b, \beta \delta\right) \\
W\left(\sigma, U_{j}^{f} \mid b, \beta \delta\right) & \equiv u(b)-c(\sigma)+\beta \delta\left\{\lambda(\sigma) E_{F}\left\{\max \left(V_{j}^{f}(w), U_{j}^{f}\right)\right\}+(1-\lambda(\sigma)) U_{j}^{f}\right\}
\end{aligned}
$$

\footnotetext{
${ }^{9}$ Appendix A of PDV provides a formal proof in the absence of a job search monitoring scheme.
} 
where $j=s$ for a sophisticated agent, $j=n$ for a naive agent and $j=e$ for an agent with exponential time preferences, in which case $\beta=1$. $B_{j}^{c}(\bar{\sigma})$ (resp., $B_{j}^{f}(\bar{\sigma})$ ) denotes the expected lifetime utility of a current (resp. future) unemployed self who complies with the job search requirement: $\sigma \geq \bar{\sigma}$ (resp., $\sigma<\bar{\sigma}$ ). In the sequel, we will write $B_{j}^{i}$ instead of $B_{j}^{i}(\bar{\sigma})$ for $i \in\{c, f\}$, except if we explicitly consider the case in which the search requirement binds. $\tilde{Z}_{j}^{c}$ is the expected lifetime utility of a noncomplying unemployed current self, whereas $Z_{j}^{f}$ is the intertemporal pay-off of a future unemployed self who is sanctioned, because he did not comply with the search requirement in the current period. To avoid repetitions, we use in (4) and in the sequel the symbol $U_{j}^{f}$ to designate either $B_{j}^{f}$ or $Z_{j}^{f}$, as the case may be. $V_{j}^{f}(w)$ is the expected lifetime utility of a future self employed in an occupation paying $w$ from the perspective of the current self.

The interpretation is as follows. The expected lifetime utility of the unemployed current self is equal to the instantaneous net utility $u(b)-c(\sigma)$ plus the discounted expected lifetime utility in the subsequent period. If the agent is a hyperbolic discounter the value of the future is discounted additionally by the short-term discount factor $\beta \leq 1$. In the subsequent period the unemployed is offered with probability $\lambda(\sigma)$ a job yielding $V_{j}^{f}(w)$ if he accepts. Otherwise he remains unemployed. In this case the continuation payoff depends on whether the current self complied or not and on whether the current self believes that the future self will comply or not to the job search requirement $\bar{\sigma}$. If the current self did not comply, we assume that he is aware that he will be sanctioned in the next period, so that the continuation value is $Z_{j}^{f}$. If the current self did comply, then he is not sanctioned in the next period. Moreover, in that case he believes that he will comply in the next period, so that the continuation value is $B_{j}^{f}(\bar{\sigma})$. This is because he acts in a stationary environment and because he therefore believes that he will set search effort in future periods at least at the same level as in the current period. Indeed, if he is a sophisticated agent, then he is aware that he is impatient, so that he is aware that his future self will set the search effort at the same level as the current self. If he is a naive agent, then he believes that his future self will behave like an exponential agent and will therefore set search effort at a higher level than the one set by the current self. Finally, the unemployed worker will comply or not, depending on which yields the highest expected lifetime utility of the current self: $\tilde{\Omega}_{j}^{c}=\max _{j}\left\{B_{j}^{c}(\bar{\sigma}), \tilde{Z}_{j}^{c}\right\}$. Note that the latter decision clearly depends on the value of the short-term discount factor $\beta$.

The optimization problem of the current self is a function of the continuation payoffs of the future selves. Since the intertemporal values of the future selves are viewed from the perspective of the current self, they are all discounted by a factor $\delta$ instead of $\beta \delta$. Consequently, for $j \in\{s, n, e\}$, first:

$$
V_{j}^{f}(w)=u(w)+\delta\left[(1-q) V_{j}^{f}(w)+q U_{j}^{f}\right]
$$

The expected lifetime utility of being employed is equal to the instantaneous utility of wage income plus the discounted benefit of the continuation payoff in the next period. With probability $1-q$ the agent remains employed, whereas with probability $q$ he is laid off. In case of layoff, we assume 
that, if the individual was never sanctioned in the past, eligibility to UB is restored irrespectively of the length of the employment spell ${ }^{10}$ By contrast, we assume that a dismissed worker is not entitled to UB if the individual did not comply with the search requirements and was sanctioned in the past. In other words, we ignore the "entitlement effect". We make this assumption, since it is not essential and since this simplifies the derivations. Note that these assumptions are implicit in the continuation payoff in case of layoff in (5), i.e. in $U_{j}^{f}$. Recall that $U_{j}^{f}$ designates either $B_{j}^{f}$ if the agent complies in all periods and $Z_{j}^{f}$ if he does not comply.

Second, the intertemporal value of the unemployed future selves depend on whether the agent is naive or sophisticated. A naive agent incorrectly believes that in the future he will exert as much search effort as an agent with an exponential discount factor. Therefore,

$$
U_{n}^{f}=U_{e}^{f}=W\left(\sigma_{e}^{U}, U_{e}^{f} \mid y^{U}, \delta\right)
$$

where $y^{U} \equiv b$ if $U_{j}^{f}=B_{j}^{f}$ for $j \in\{n, s, e\}$, i.e. if the agent complies with the job search requirement; $y^{U} \equiv z$ if $U_{j}^{f}=Z_{j}^{f}$ for $j \in\{n, s, e\}$, i.e. if he does not comply; $\sigma_{e}^{U}=\sigma_{e}^{B} \equiv$ $\arg \max _{\sigma \geq \bar{\sigma}} W\left(\sigma, B_{e}^{f} \mid b, \delta\right)$ if $U_{e}^{f}=B_{e}^{f}$, i.e. the optimal search effort of an exponential agent who complies; $\sigma_{e}^{U}=\sigma_{e}^{Z} \equiv \arg \max _{\sigma} W\left(\sigma, Z_{e}^{f} \mid z, \delta\right)$ if $U_{e}^{f}=Z_{e}^{f}$, i.e. the optimal search effort of an exponential agent who does not comply.

By contrast, a sophisticated agent realizes that he will procrastinate in the future and sets search effort like the current self:

$$
U_{s}^{f}=W\left(\sigma_{s}^{U}, U_{s}^{f} \mid y^{U}, \delta\right)
$$

where $\sigma_{s}^{U}=\sigma_{s}^{B} \equiv \arg \max _{\sigma \geq \bar{\sigma}} W\left(\sigma, B_{s}^{f} \mid b, \beta \delta\right)$ if $U_{s}^{f}=B_{s}^{f}$ and $\sigma_{s}^{U}=\sigma_{s}^{Z} \equiv \arg \max _{\sigma} W\left(\sigma, Z_{s}^{f} \mid\right.$ $b, \beta \delta)$. Consequently, short-run impatience $(\beta<1)$ also reduces the continuation values of the future selves of sophisticated agents relatively to naive agents. This follows immediately from the fact that, in contrast to the future selves of a naive agent, the future selves of a sophisticated agent does not set search effort to maximize lifetime utility of his future selves.

\subsection{The First Order Conditions of the Optimization Problem}

The optimization problem set up in the previous subsection involves three decision variables: (i) the decision to comply or not, (ii) the decision how much to search and (iii) the decision whether or not to accept job offers. The optimal choice with respect to the first decision variable concerns a discrete choice and cannot therefore be characterized by a first order condition in which the cost and benefit of the decision variable should balance. By contrast, the choices with regards the two other decision variables can be characterized by first order conditions. In this subsection we characterize

\footnotetext{
${ }^{10}$ This is a simplifying assumption, as in reality the entitlement to UB usually depends on the past record of insurance contributions while employed. However, our assumption is not too restrictive: Paserman (2008) shows that the findings do not crucially depend on this assumption.
} 
these conditions. In the subsequent section we show how we can use these conditions together with the compliance decision to characterize the behavior of unemployed workers with hyperbolic time preferences who are entitled to UB subject to job search requirements.

First, we show that the optimal job acceptance decision is equivalent to setting a reservation wage such that the marginal benefit of increasing it is equal to the marginal cost. Subsequently, we derive the first order condition of job search effort. We do this for both, the case that the current and future selves comply $\left(U_{j}^{f}=B_{j}^{f}\right)$ and the case that they do not comply with the job requirement $\bar{\sigma}\left(U_{j}^{f}=Z_{j}^{f}\right)$.

Using (5), we can write

$$
V_{j}^{f}(w)-U_{j}^{f}=\frac{u(w)-(1-\delta) U_{j}^{f}}{1-\delta(1-q)}
$$

It is easily seen that with the stated assumptions this function strictly increasing in $w$. Consequently, this function defines a unique reservation wage $x_{j}^{U}$ for $j \in\{n, s, e\}$ and $U \in\{B, Z\}$ such that $V_{j}^{f}\left(x_{j}^{U}\right)-U_{j}^{f} \equiv 0$ and $V_{j}^{f}(w)>U_{j}^{f}\left(V_{j}^{f}(w)<U_{j}^{f}\right)$ if and only if $w>x_{j}^{U}\left(w<x_{j}^{U}\right)$ :

$$
u\left(x_{j}^{U}\right) \equiv(1-\delta) U_{j}^{f}
$$

Using this reservation wage property, we can rewrite $W\left(\sigma, U_{j}^{f} \mid y^{U}, \beta \delta\right)$ as follows (see Appendix A):

$$
W\left(\sigma, U_{j}^{f} \mid y^{U}, \beta \delta\right)=u\left(y^{U}\right)-c(\sigma)+\beta \delta\left\{\frac{\lambda(\sigma)}{1-\delta(1-q)} Q\left(x_{j}^{U}\right)+U_{j}^{f}\right\}
$$

where

$$
Q\left(x_{j}^{U}\right) \equiv \int_{x_{j}^{U}}^{\bar{w}}[u(w)-u(x)] d F(w)
$$

Inserting this expression for $\beta=1$ in (6) and (7) and using (9) to take the dependence of the reservation wage on $U_{j}^{f}$ into account yields the first order conditions of the reservation wage for $j \in\{n, s, e\}:$

$$
\begin{aligned}
& u\left(y^{U}\right)+\frac{\delta \lambda\left(\sigma_{e}^{U}\right)}{1-\delta(1-q)} Q\left(x_{j}^{U}\right)=c\left(\sigma_{e}^{U}\right)+u\left(x_{j}^{U}\right) \text { for } j \in\{n, e\} \\
& u\left(y^{U}\right)+\frac{\delta \lambda\left(\sigma_{s}^{U}\right)}{1-\delta(1-q)} Q\left(x_{s}^{U}\right)=c\left(\sigma_{s}^{U}\right)+u\left(x_{s}^{U}\right)
\end{aligned}
$$

where $x_{n}^{U}=x_{e}^{U}$.

The left-hand side of (12) is equal to the benefit of continuing search one more period rather than accepting a job offer at the reservation wage. This is the instantaneous utility of income when unemployed, with or without entitlement to UB, plus the expected discounted wage gain in case of continued search. This is equal to the cost of continuing search, as expressed by the right-hand side of (12). This is the instantaneous disutility of job search plus the foregone instantaneous utility of accepting a job offer at the reservation wage. 
Inserting (10) in (2) and (3) and differentiating with respect to $\sigma$, yields the first order conditions of job search effort for $j \in\{n, s, e\}$. For $\mu_{j}^{U} \geq 0$ :

$$
\frac{\beta \delta \lambda^{\prime}\left(\sigma_{j}^{U}\right)}{1-\delta(1-q)} Q\left(x_{j}^{U}\right)+\mu_{j}^{U}=c^{\prime}\left(\sigma_{j}^{U}\right) \text { and } \mu_{j}^{U}\left(\sigma_{j}^{U}-\bar{\sigma}\right)=0
$$

where $\mu_{j}^{U} \geq 0$ is the Lagrange multiplier associated to the inequality constraint $\sigma_{j}^{U} \geq \bar{\sigma}$.

This equation states that in the optimum the marginal benefit of search should equal its marginal cost, unless the constraint is binding. If the constraint is binding, $\sigma_{j}^{U} \geq \bar{\sigma}$ and marginal cost of search exceeds the marginal benefit. The agent would then like to decrease search effort, but cannot, since he would then violate the job search requirement. Note also that if the agent does not comply $\mu_{j}^{U}=\mu_{j}^{Z}=0$, since the inequality constraint can then not bind.

\section{A Graphical Representation of the Solution}

In the previous subsection we characterized the solution of the optimization problem by the first order conditions of the reservation wage and the job search effort chosen by agents with hyperbolic time preferences. However, we did not yet discuss the properties of this solution. In particular, we did not describe how the solution changes with the way in which agents discount the future. This is what we do in this section. We first describe the solution for a naive agent and, subsequently, for a sophisticated agent. Since the solution of the naive agent depends on the solution of an agent with exponential time preferences, a comparison with the latter is quite naturally integrated in this presentation.

This graphical characterization of the solution fosters a more intuitive understanding of results proven by PDV. In addition, it helps to explain how the solution changes as job search requirements are imposed through a job search monitoring scheme, a complication ignored in the analysis of PDV. In particular, it clearly explains the point at which agents stop complying to the search requirement and how this point is affected by the time preferences of agents. It will be shown that, as for the decision regarding job search intensity, the decision to comply or not involves a conflict between the current and future selves. Finally, the graphical analysis suggests a simpler way of proving uniqueness of the solution for all types of agents than in PDV (see the proof of their Theorem 1), which we generalize for risk averse agents and for the presence of job search requirements. We close this section by this proof.

In Subsection 3.3 we characterized the optimal reservation wage $x_{j}^{U}$ and job search effort $\sigma_{j}^{U}$ for $j \in\{n, s, e\}$ by means of first order conditions (12) and 13). In the graphical analysis that we present in this section we will plot these first order conditions in the $(\sigma, x)$-space, so that the intersections of these curves pinpoint the optimal solutions. To this purpose, we define the implicit functions $R\left(\sigma, x \mid y^{U}\right)=0$ and $S(\sigma, x \mid \beta)=0$ to represent the interior solutions of these first order 
conditions as a function of $\sigma$ and $x$ for $\beta \leq 1$ :

$$
\begin{aligned}
R\left(\sigma, x \mid y^{U}\right) & \equiv u\left(y^{U}\right)+\frac{\delta \lambda(\sigma)}{1-\delta(1-q)} Q(x)-c(\sigma)-u(x)=0, \\
S(\sigma, x \mid \beta) & \equiv \frac{\beta \delta \lambda^{\prime}(\sigma)}{1-\delta(1-q)} Q(x)-c^{\prime}(\sigma)=0
\end{aligned}
$$

From these equations it is immediately clear that the short term discount factor $\beta$ only affects search effort directly and the reservation wage only indirectly via the choice of the job search effort, a point that was already stressed by PVD and Paserman (2008). Furthermore, the decision to comply or not to the job search requirements only shifts the first order condition of the reservation wage. If the unemployed worker complies, $U_{j}^{f}=B_{j}^{f}$ and therefore $y^{U} \equiv b$. If he does not comply, $U_{j}^{f}=Z_{j}^{f}$ and therefore $y^{U} \equiv z . S(\sigma, x \mid \beta)$ is not affected. These points are formally shown in Proposition 1 (iii) and (iv) below.

In Proposition 1 (i) and (ii) it is shown that the implicit function $S(\sigma, x \mid \beta)=0$ is always decreasing in the $(\sigma, x)$-space and $R\left(\sigma, x \mid y^{U}\right)=0$ increasing (decreasing) for all $\sigma$ smaller (bigger) than the optimal job search effort of an exponential agent, i.e. $\sigma_{e}^{U}$. The intuition of the first result is as follows. Since the job arrival rate displays decreasing returns and the net disutility of job search increasing marginal costs, the net marginal return to job search effort is a decreasing function of $\sigma$ for any given reservation wage $x$. Since the likelihood of finding a wage above the reservation wage declines with the reservation wage $\left(Q^{\prime}(x)<0\right)$, the expected gain of intensified search falls with the reservation wage. Therefore, since along $S(\sigma, x \mid \beta)=0$ the net marginal return to job search needs to remain equal to zero, the reservation wage must decrease as $\sigma$ increases. This curve will therefore be steeper the costlier is job search for an individual.

The second result means that the reservation wage and, hence, by (9), the intertemporal utility of a future self are maximized if job search effort is set at the level that is optimal for the exponential agent, i.e. at a level that is optimal where only the long-run discount factor $\delta$ matters. This makes sense, since only the long-run discount factor $\delta$ matters for setting the reservation wage. Setting $\sigma$ below or above $\sigma_{e}^{U}$ must therefore necessarily lower lifetime utility and, hence, the reservation wage. It follows that $R\left(\sigma, x \mid y^{U}\right)=0$ must be hump-shaped and reach a maximum where it crosses the $S(\sigma, x \mid 1)=0$ curve. The steeper $S(\sigma, x \mid 1)=0$, the more concave is this curve.

\section{Proposition 1.}

Let $x=r\left(\sigma \mid y^{U}\right)$ and $x=s(\sigma \mid \beta)$ denote the explicit functions corresponding to the implicit functions $R\left(\sigma, x \mid y^{U}\right)=0$ and $S(\sigma, x \mid \beta)=0, h\left[\sigma, r\left(\sigma \mid y^{U}\right)\right] \equiv \lambda(\sigma) \bar{F}\left[r\left(\sigma \mid y^{U}\right)\right]$ the instantaneous probability of leaving unemployment for employment, and $\bar{F}[.] \equiv 1-F[$.$] .$ Then $\forall \beta \in(0,1], \forall y \in \mathbb{R}^{+}$and

(i) $\forall \sigma \in \mathbb{R}^{+}: \frac{\partial \log s(\sigma \mid \beta)}{\partial \log \sigma}=\left[\frac{\partial \log Q[s(\sigma \mid \beta)])}{\partial \log s(\sigma \mid \beta)}\right]^{-1}\left(\frac{d \log c^{\prime}(\sigma)}{d \log \sigma}-\frac{d \log \lambda^{\prime}(\sigma)}{d \log \sigma}\right)<0$, 
(ii) $\forall \sigma \lesseqgtr \sigma_{e}^{U}: \frac{\partial r\left(\sigma \mid y^{U}\right)}{\partial \sigma}=S\left[\sigma, r\left(\sigma \mid y^{U}\right) \mid 1\right] \frac{1-\delta(1-q)}{\left[1-\delta(1-q)+\delta h\left[\sigma, r\left(\sigma \mid y^{U}\right)\right]\right] u^{\prime}\left[r\left(\sigma \mid y^{U}\right)\right]} \gtreqless 0$,

(iii) $\forall \sigma \in \mathbb{R}^{+}: \frac{\partial s(\sigma \mid \beta)}{\partial \beta}>0$ and $\frac{\partial s(\sigma \mid \beta)}{\partial y^{U}}=0$,

(iv) $\forall \sigma \in \mathbb{R}^{+}: \frac{\partial r\left(\sigma \mid y^{U}\right)}{\partial y^{U}}>0$ and $\frac{\partial r\left(\sigma \mid y^{U}\right)}{\partial \beta}=0$.

Proof. See Appendix B.

\subsection{The Naive Agent}

We first consider the solution of the naive agent. In Section 3.3 we have shown that the naive agent will first set the reservation wage at the level at which the exponential agent would set it. Therefore, in order to determine this reservation wage, we must first solve the optimization problem of the exponential agent. We start by considering the problem for an agent who complies $\left(U_{e}^{f}=B_{e}^{f}\right)$ and for whom the job search requirement $\bar{\sigma}$ does not bind, so that we can consider the interior solution $\left(\sigma_{e}^{B}, x_{e}^{B}\right)$. This solution can be found by solving for $x$ and $\sigma$ from the first order conditions (14) and (15) in which $\beta=1$ and $y^{U}=y^{B}=b$. Graphically, this corresponds to the intersection of the curves $r(\sigma \mid b)$ and $s(\sigma \mid 1)$ represented by point A in Figure 1. Note that, in line with Proposition 1 (ii), this intersection occurs at the maximum of $r(\sigma \mid b)$. The naive agent then sets job search effort such that it solves the first order condition (15) for $\beta<1$ and for $x=x_{e}^{B}=x_{n}^{B}$. Graphically, $\left(\sigma_{n}^{B}, x_{n}^{B}\right)$ can therefore be found at point B in Figure 1.

Consider now the optimal solution of a naive agent who does not comply $\left(U_{e}^{f}=Z_{e}^{f}\right)$. Using Proposition 1 (iii) and (iv) and the fact that $y^{B}=b>z=y^{Z}$, one obtains that $r(\sigma \mid z)<r(\sigma \mid b)$ and that $s(\sigma \mid \beta)$ does not depend on whether or not the agent complies. Consequently, following the same line of arguments as for the complying agent, one can find that $\left(\sigma_{e}^{Z}, x_{e}^{Z}\right)$ corresponds to point $\mathrm{C}$ and $\left(\sigma_{n}^{Z}, x_{n}^{Z}\right)$ to point $\mathrm{D}$ in Figure 1. Clearly, if the job search requirement $\bar{\sigma}$ does not bind, the reservation wage and, hence the expected lifetime utility, is higher for the agent who complies: $x_{n}^{B}>x_{n}^{Z}$ and, by (9), $B_{n}^{c}>\tilde{Z}_{n}^{c}$.

Suppose now that the job search requirement binds if the agent complies, so that search effort equals $\bar{\sigma}>\sigma_{n}^{B}$ and the first order condition with respect to search is no longer satisfied. The BAF curve displays the evolution of the reservation wage when $\bar{\sigma}$ increases. By $(9)$, the expected lifetime utility of the complying future self, i.e. $B_{n}^{f}(\bar{\sigma})$, evolves according to the BAF curve. Observe that initially (from B up to A) a reinforcement of the job search requirement does not affect the welfare of the future self of the naive agent, since he falsely believes that he will behave like an exponential agent in the future who sets job search above the job search requirement: $\sigma_{e}^{B}>\bar{\sigma}$. To the right of point $\mathrm{A}$, the job search requirement starts to bind for an exponential agent as well, so that the welfare of the future self starts decreasing. The welfare of the complying current self is, however, immediately negatively influenced by a strengthening of the search requirement (see the downward-sloping curve starting at point B'). This is because, as formally demonstrated in the 


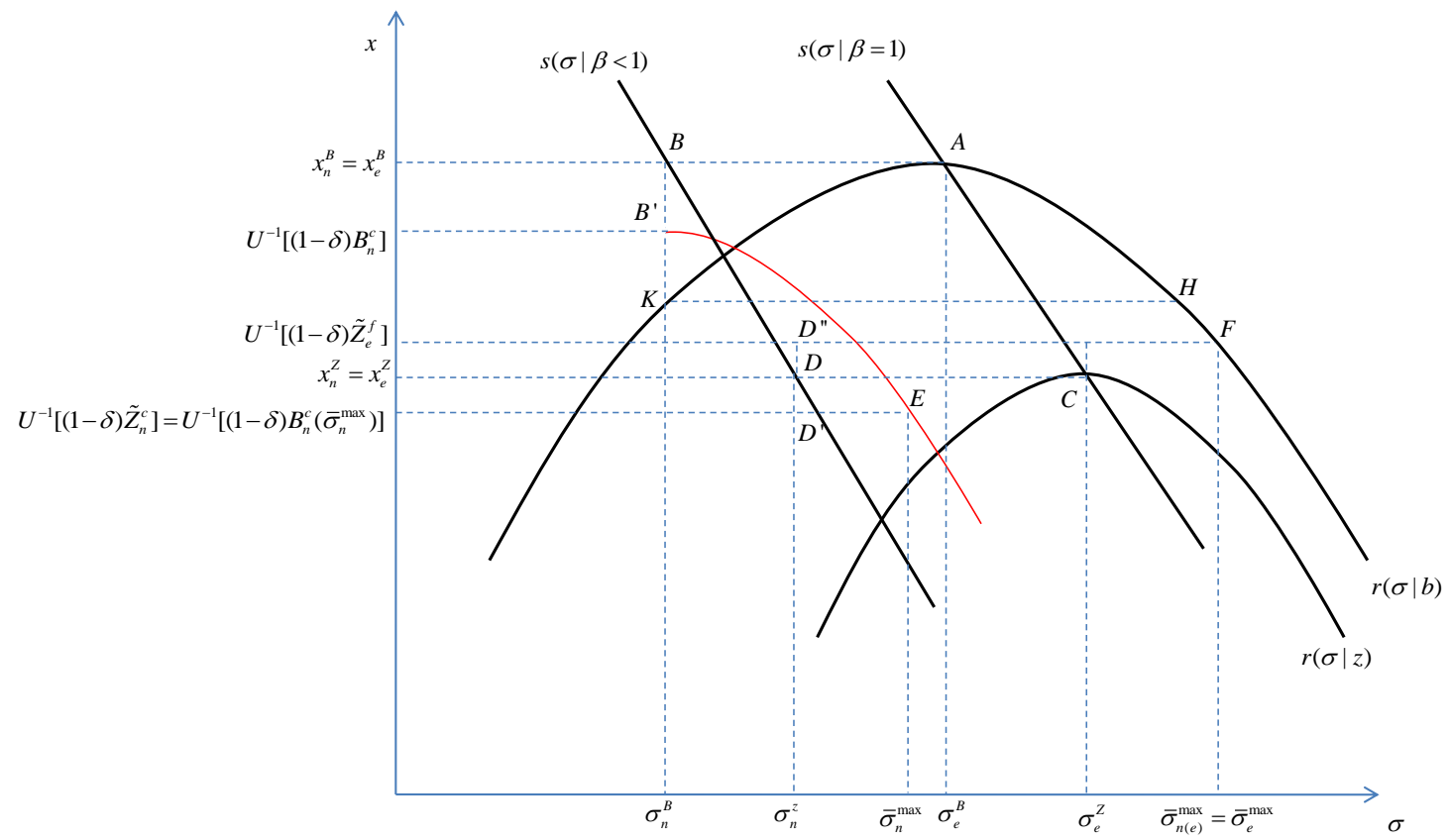

Figure 1: The Solution for the Naive Agent in Case of Perfect Monitoring. $x=$ reservation wage; $\sigma=$ realized search effort.

proof of Proposition 2 (ii) (a) below, as $\bar{\sigma}$ starts binding the instantaneous cost of search increases, while the reservation wage remains unaffected.

A similar analysis can be conducted to find the optimal solution of a non complying exponential agent at point $\mathrm{C}$ and of a naive agent at point $\mathrm{D}$ in Figure 1. If the job search requirement is set at a too high level, an initially complying unemployed worker may decide to stop complying. This occurs if $\bar{\sigma}$ increases beyond $\bar{\sigma}_{n}^{\max }$, a level of search intensity at which the current self is indifferent between complying or not, implicitly defined by $B_{n}^{c}\left(\bar{\sigma}_{n}^{\max }\right) \equiv \tilde{Z}_{n}^{c} 11$ In Figure 1, it corresponds to the search intensity attained at point $\mathrm{E}$.

Without choosing particular functional forms and parameters we can say little about the exact level of $\bar{\sigma}_{n}^{\max }$. Nevertheless, in Proposition 2 (iii) (a) it is demonstrated that we can bracket its level: $\bar{\sigma}_{n}^{\max } \in\left(\sigma_{n}^{Z}, \bar{\sigma}_{n(e)}^{\max }\right)$, where $B_{n}^{f}\left(\bar{\sigma}_{n(e)}^{\max }\right)=\tilde{Z}_{n}^{f}$ the latter measuring the expected lifetime utility of a non-complying future self (i.e. $\tilde{Z}_{n}^{f} \equiv Z_{n}^{f}+u(b)-u(z)>Z_{n}^{f}$ ). Since $\bar{\sigma}_{n(e)}^{\max }=\bar{\sigma}_{e}^{\max }$ (point $\mathrm{F}$

\footnotetext{
${ }^{11}$ Note that, by Proposition 2 (i) (b), we cannot exclude that $\tilde{Z}_{n}^{c}$ is larger than $Z_{n}^{f}$. This can happen if $\beta$ is close to one, so that the fact that the non-complying current self earns during one period $b$ rather than $z$ dominates the higher discounting of future utility. In Figure 1 it is assumed that $\beta$ is sufficiently low so that $\tilde{Z}_{n}^{c}<Z_{n}^{f}$.
} 
in Figure 1), the naive agent stops complying at a lower level of search effort than at which an exponential agent would do so. This means that the issue that too high job search requirements induce exits from the claimant status (Manning, 2009, Petrongolo, 2009), is even more important for impatient agents. We return to this point in Section 5 where we discuss the welfare implications of the introduction of a monitoring scheme.

\section{Proposition 2.}

(i) For $j \in\{n, s\}$ :

(a) $\forall \bar{\sigma} \in \mathbb{R}^{+}: B_{j}^{f}(\bar{\sigma})>B_{j}^{c}(\bar{\sigma})$.

(b) $\tilde{Z}_{j}^{f} \lesseqgtr \tilde{Z}_{j}^{c} \Leftrightarrow \beta \gtreqless 1-\frac{u(b)-u(z)}{\delta\left\{\frac{\lambda\left(\sigma_{j}^{z}\right)}{1-\delta(1-q)} Q\left(x_{j}^{z}\right)+Z_{j}^{f}\right\}}$.

(c) $\forall \bar{\sigma}<\sigma_{e}^{U}: U_{n}^{c}(\bar{\sigma})>U_{s}^{c}(\bar{\sigma})$ and $\forall \bar{\sigma} \geq \sigma_{e}^{U}: U_{n}^{c}(\bar{\sigma})=U_{s}^{c}(\bar{\sigma})$ where $U \in\{B, Z\}$.

(ii) (a) $\frac{\partial B_{n}^{c}(\bar{\sigma})}{\partial \bar{\sigma}}=0$ iff $\bar{\sigma} \leq \sigma_{n}^{B}$ and $\frac{\partial B_{n}^{c}(\bar{\sigma})}{\partial \bar{\sigma}}<0$ iff $\bar{\sigma}>\sigma_{n}^{B}$.

(b) $\exists \sigma_{s}^{*} \in\left(\sigma_{s}^{B}, \sigma_{e}^{B}\right)$ such that $\forall \bar{\sigma} \lesseqgtr \sigma_{s}^{*}$ and $\bar{\sigma}>\sigma_{s}^{B}: \frac{\partial \tilde{B}_{s}^{c}(\bar{\sigma})}{\partial \bar{\sigma}} \gtreqless 0$.

(iii) For $j \in\{n, s\}$, let $\bar{\sigma}_{j(e)}^{\max }$ denote the threshold value at which the UB claimant would stop complying if he would have taken this decision on the basis of a utility function in which the future is discounted at an exponential rate, i.e. implicitly defined by $B_{j}^{f}\left(\bar{\sigma}_{j(e)}^{\max }\right)=\tilde{Z}_{j}^{f} \equiv$ $Z_{j}^{f}+u(b)-u(z)>Z_{j}^{f}$. Then the level of search requirement above which the unemployed prefers to withdraw from the UB claimant register, $\bar{\sigma}_{j}^{\max }$, verifies:

(a) $\bar{\sigma}_{n}^{\max } \in\left(\sigma_{n}^{Z}, \bar{\sigma}_{n(e)}^{\max }\right)$,

(b) $\bar{\sigma}_{s}^{\max } \in\left(\max \left\{\sigma_{s}^{Z}, \tilde{\sigma}_{s}\right\}, \bar{\sigma}_{s(e)}^{\max }\right)$

where $\tilde{\sigma}_{s}$ solves $B_{s}^{c}\left(\tilde{\sigma}_{s}\right)=B_{s}^{c}$, the latter designating the unconstrained intertemporal utility of the current self, and $\tilde{\sigma}_{s}>\sigma_{s}^{*}>\sigma_{s}^{B}$.

Proof. See Appendix C.

\subsection{The Sophisticated Agent}

Let us now consider the behaviour of a sophisticated agent who complies $\left(U_{s}^{f}=B_{s}^{f}\right)$ and for whom the job search requirement $\bar{\sigma}$ does not bind, so that we can consider the interior solution $\left(\sigma_{s}^{B}, x_{s}^{B}\right)$. This solution can be found by solving for $x$ and $\sigma$ from the first order conditions (14) and (15) in which $\beta<1$ and $y^{U}=y^{B}=b$. Graphically, this corresponds to the intersection of the curves $r(\sigma \mid b)$ and $s(\sigma \mid \beta<1)$ represented by point $\mathrm{O}$ in Figure 2. Since the sophisticated agent, contrary to the naive agent, takes into account that he will procrastinate in the subsequent period, his reservation wage will be lower and his search intensity will be higher than that of the naive agent (point B), 


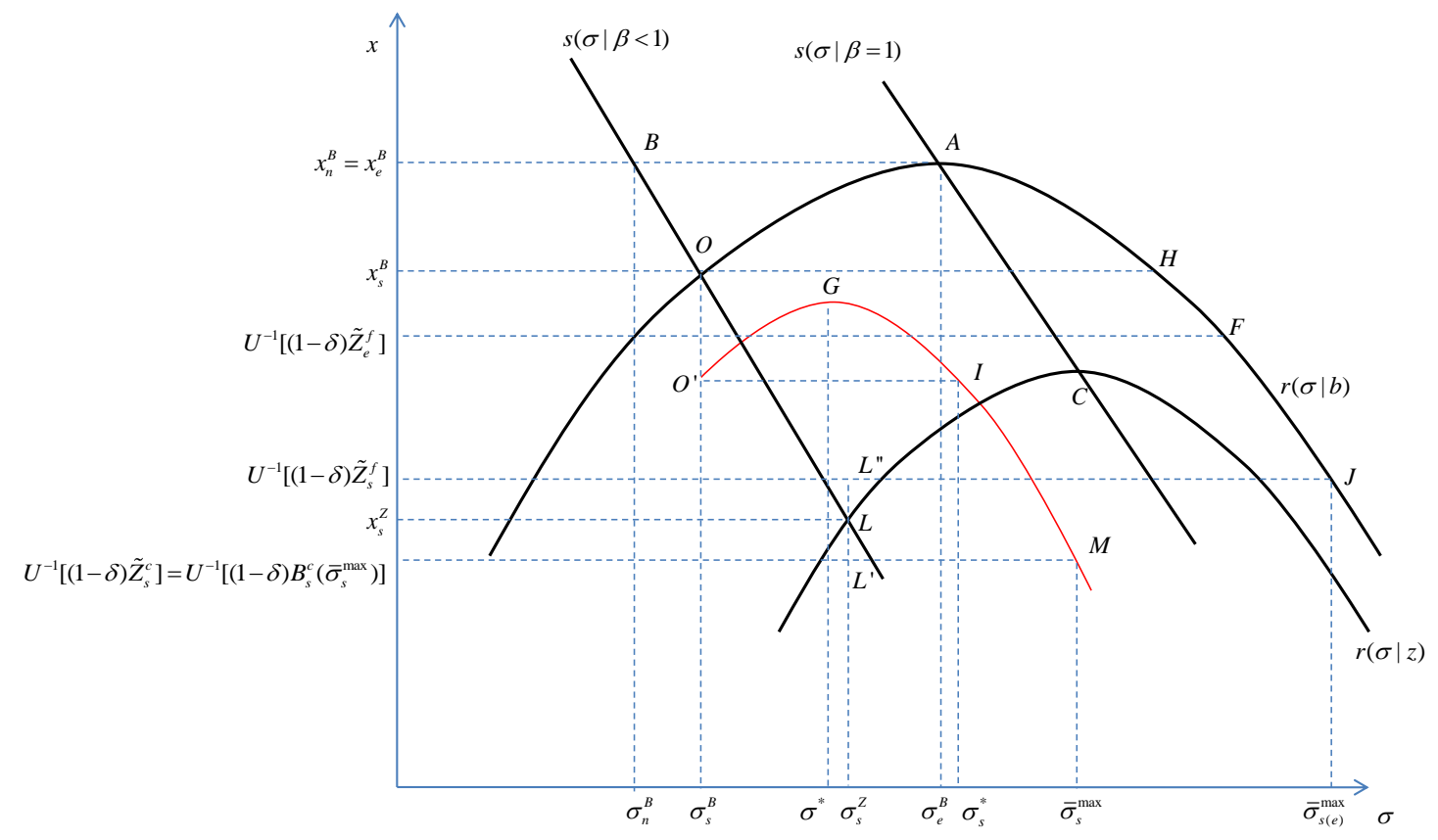

Figure 2: The Solution for the Sophisticated Agent in Case of Perfect Monitoring. $x=$ reservation wage; $\sigma=$ search effort.

but it is still lower than that of an exponential agent (point A). Following a similar reasoning, we find that the sophisticated agent who does not comply chooses point L in Figure 2.

As we increase the job search requirement $\bar{\sigma}$ above $\sigma_{s}^{B}$, it starts to bind. Contrary to the naive agent, the sophisticated one knows that he has a self-control problem, so that the expected lifetime utility of the future self (and hence the reservation wage) strictly increases with the requirement up to $x_{e}^{B}$ (at point A) and strictly decreases afterwards. However, the decision to comply depends on the expected lifetime utility of the current self. This utility also initially increases with the requirement starting from $\sigma_{s}^{B}$, because current utility depends on future utility and because $\sigma_{s}^{B}$ is optimally chosen from the perspective of the current self, so that close to the right of $\sigma_{s}^{B}$ the marginal cost of search is only slightly higher than the marginal benefit. However, as the search requirement increases further, the net marginal cost of search increases further while the expected utility of the future self decreases at a decreasing rate as search effort approaches the optimal level $\sigma_{e}^{B}$ of the exponential agent. In Proposition 2 (ii) (b) it is indeed formally shown that the expected lifetime utility of the current self attains a maximum strictly between $\sigma_{s}^{B}$ and $\sigma_{e}^{B}$. The inverse U-curve in Figure 2 with a maximum at $\mathrm{G}$ represents therefore the expected lifetime utility of the 
complying current self.

The sophisticated agent complies with the search requirement until the lifetime utility of the complying current self does not fall below that of the non-complying current self, i.e. as long as $\bar{\sigma}<\bar{\sigma}_{s}^{\max }$ (to the left of point $\mathrm{M}$ ), where $B_{s}^{c}\left(\bar{\sigma}_{s}^{\max }\right)=\tilde{Z}_{s}^{c}$. As for the naive agent, we cannot in general be very precise about the level of $\bar{\sigma}_{s}^{\max }$, but in Proposition 2 (iii) (b) it is again shown that we can bracket this threshold in an interval: $\bar{\sigma}_{s}^{\max } \in\left(\sigma_{s}^{Z}, \bar{\sigma}_{s(e)}^{\max }\right)$ where $B_{s}^{f}\left(\bar{\sigma}_{s(e)}^{\max }\right)=\tilde{Z}_{s}^{f} \equiv$ $Z_{s}^{f}+u(b)-u(z)>Z_{s}^{f}$. Compared to the naive agent, this interval is shifted to the right, so that non-compliance is a somewhat less important issue than for a naive agent. Since $\bar{\sigma}_{e}^{\max }<\bar{\sigma}_{s(e)}^{\max }$, we cannot exclude that a sophisticated agent stops complying at a higher search intensity (point J) than an exponential agent (point F).

Observe that $\bar{\sigma}_{s}^{\max }$ should be not only larger than $\sigma_{s}^{Z}$, but also bigger than $\tilde{\sigma}_{s}$, where $B_{s}^{c}\left(\tilde{\sigma}_{s}\right)=B_{s}^{c}$ (point I). For, if the search requirement is set at (to the left of) $\tilde{\sigma}_{s}$ the complying current self attains the same (more) utility as (than) what he obtains if he decides about the level of job search intensity without any constraint. This utility can never be lower than his free choice, $\sigma_{s}^{Z}$, if he does not comply and therefore he will never stop complying if $\bar{\sigma} \in\left[\sigma_{s}^{B}, \tilde{\sigma}_{s}\right]$. In sum, $\bar{\sigma}_{s}^{\max } \in\left(\max \left\{\sigma_{s}^{Z}, \tilde{\sigma}_{s}\right\}, \bar{\sigma}_{s(e)}^{\max }\right)$.

\subsection{Uniqueness of the Solution}

Finally, we show that with the given assumptions these solutions for both the naive and the sophisticated agent are unique. This is a generalization of Theorem 1 of PDV, who prove this for risk neutral unemployed who are not subject to job search requirements.

Proposition 3. For both the sophisticated and the naive agent, the solution is unique.

Proof. See Appendix D.

\section{Can Monitoring Search Effort Be Socially Efficient?}

As explained above, job-seekers with time-consistent preferences will always loose if binding job search requirements are imposed. Proposition 1 of PDV shows that a marginal increase above the freely chosen search effort of a sophisticated agent raises the utility of the current self. For the same type of agent, Paserman (2008) provides simulation results where monitoring job search improves worker's long-run utility, reduce unemployment duration and lower government expenditures. In comparison with these earlier properties, we provide analytical results that are not restricted to marginal changes and are valid under risk aversion. First, in Proposition 5 we demonstrate that imposing binding search requirements always increases the exit rate from unemployment to employment for a naive agent and under mild conditions on the wage offer distribution and the utility function for the sophisticated agent. This shows that the findings of Paserman (2008) also hold for 
risk averse agents and provides precise conditions under which these findings can be generalized ${ }^{12}$ Second, we discuss the implications of this result on social welfare. In particular, we discuss the conflict that stricter job search requirements induce between the current self and the future selves. Even if we accept that from a normative point of view we should only care about the preferences of an agent with a long-run perspective, and, hence, of the future self, we argue that this conflict may inhibit the implementation of socially efficient job search requirement. The reason is that the decision to comply depends on the utility of the current self. The conflict between the current and future self may therefore imply that a socially efficient solution cannot be attained by the monitoring of job search. A consequence is that other policies that do not induce a conflict between the current self and the future selves, such as job search assistance, may potentially be socially more efficient than imposing stricter job search requirements. However, whether such alternative policy is indeed more socially efficient depends on its effectiveness in raising job search effort and on its implementation costs relative to a monitoring scheme.

\subsection{How Do Stricter Job Search Requirements Affect the Exit Rate From Un- employment?}

In this section we show under which conditions imposing job search requirements above the free choices of naive and sophisticated agents, i.e. above $\sigma_{n}^{B}$ and $\sigma_{s}^{B}$, increases the exit rate from unemployment to employment.

Proposition 4. For a naive agent the exit rate from unemployment always strictly increases with the job search requirement.

Proof. For a naive agent, the exit rate from unemployment for any $\bar{\sigma} \in\left[\sigma_{n}^{B}, \sigma_{e}^{B}\right]$ is given by $h\left(\bar{\sigma}, x_{e}^{B}\right)=\lambda(\bar{\sigma}) \bar{F}\left(x_{e}^{B}\right)$. Since the reservation wage is fixed at $x_{e}^{B}$ and $\lambda(\bar{\sigma})$ is strictly increasing in $\bar{\sigma}$, the exit rate must unambiguously increase. For $\bar{\sigma}>\sigma_{e}^{B}$, the exit rate is $h[\bar{\sigma}, r(\bar{\sigma} \mid b)]$. Since, by Proposition 1 (ii) the reservation $r(\bar{\sigma} \mid b)$ is decreasing with $\bar{\sigma}$ for $\bar{\sigma}>\sigma_{e}^{B}$, this decrease in the reservation wage reinforces the positive relationship between the job requirement and the exit rate.

\footnotetext{
${ }^{12}$ Our Proposition 5 are closely related to Proposition 3 of PVD in which it is shown that the exit rate from unemployment of agents with hyperbolic time preferences increases with $\beta$. However, we allow for risk aversion and demonstrate that for sophisticated agents this property holds under even milder conditions than those provided by PVD.
} 


\section{Proposition 5.}

Let $\psi(x) \equiv f(x) / \bar{F}(x)$ denote the hazard rate of the wage offer distribution, then imposing a job search requirement $\bar{\sigma}>\sigma_{s}^{B}$ on a sophisticated agent always increases the exit rate from unemployment to employment $h[\bar{\sigma}, r(\bar{\sigma} \mid b)]$ if

(1) $\forall \bar{\sigma}>\sigma_{s}^{B}: u(b) \geq c(\bar{\sigma})$ and if

(2) $\forall w \in\left(x_{s}^{B}, \bar{w}\right): \frac{d \log \psi(w)}{d \log w} \geq-\frac{d \log u(w)}{d \log w}+\frac{d \log u^{\prime}(w)}{d \log w}$.

Proof. See Appendix E.

Assumption (1) means that the cost of job search should not exceed the utility flow from income. Under risk neutrality, van den Berg (1994) makes a similar assumption, namely $0<b<\infty$ which he interprets as "...the official unemployment benefits level minus per-period search costs, and the difference of two positive variables has an indeterminate sign. However, in order to survive, individuals need a positive net (of search costs) income flow". Our assumption $u(b)-c(\sigma) \geq 0$ has the same interpretation in the context of risk aversion.

A sufficient condition for satisfying Assumption (2) is an increasing hazard rate $\psi(w)$ on $\left(x^{B}, \bar{w}\right)$. This condition is satisfied for log-concave wage offer densities 13 Among log-concave densities are the exponential and beta distributions, the families of logistic and extreme value distributions that are truncated from below at zero, uniform distributions for which the lower point support is nonnegative, normal distributions truncated at zero, Weibull distributions for which $\alpha \geq 0$, gamma distributions for which $\gamma \geq 0$ (van den Berg, 1994). However, some commonly used wage densities are not log-concave. For instance, the log-normal distribution has decreasing hazard rates for sufficiently large values and the hazard rate of a Pareto decreases on all its support van den Berg, 1994). However, these distributions satisfy the Increasing Proportionate Failure Rate (IPFR) property, which corresponds to the condition imposed in Assumption (2) under risk-neutrality:

$$
\frac{d \log \psi(w)}{d \log x} \geq-1 \Leftrightarrow w \cdot \psi(w) \text { is nondecreasing }
$$

on $\left(x_{s}^{B}, \bar{w}\right)$. The condition requires that the failure rate $\psi(w)$ does not decrease "too much" on $\left(x_{s}^{B}, \bar{w}\right)$. We refer the reader to van den Berg (1994) to find a list of other distributions that satisfy the IPFR property.

A standard assumption under risk aversion is the CRRA utility function:

$$
u(w) \equiv \frac{w^{1-\rho}}{1-\rho} \quad \text { with } \rho>0, \rho \neq 1 .
$$

Then, it can be checked that Assumption (2) of Proposition 5 is nothing else than (16), so that density functions with the IPFR property verify Assumption (2) under this specification of riskaversion.

\footnotetext{
${ }^{13}$ This is also a sufficient condition for the condition in Proposition 3 of PVD.
} 


\subsection{How Does the Imposition of Job Search Requirements Affect Social Wel- fare?}

For the sake of clarity, we first ignore the issue of non compliance. The latter is reintroduced at the end of this subsection. From the graphical analysis in Section 4 and from Propositions 1 (ii) and 2 (ii) we can easily deduce that imposing binding job search requirements initially increases welfare of both the current and the future self of a sophisticated agent. However, the range of $\bar{\sigma}$ for which Pareto improvements are attained is more restricted for the current self (up to $\sigma_{s}^{*}$ associated to point I in Figure 2) than for the future self (up to point $\mathrm{H}$ in Figure 2). For $\bar{\sigma} \in\left(\sigma_{n}^{B}, \sigma_{e}^{B}\right)$, imposing a higher search requirement does not affect the naive's long-term utility because the reservation wage stays constant between $\mathrm{B}$ and $\mathrm{A}$ in Figure 1. If $\bar{\sigma}>\sigma_{e}^{B}$, rising the requirement implies that the future self of the naive agent is worse off than in the absence of search requirements, exactly as an exponential agent. For the current self of the naive agent, utility decreases immediately once the job search requirement is set higher than $\sigma_{n}^{B}$. Based on a Pareto efficiency criterion that requires that all period selves (weakly) increase utility, imposing job search requirements above the level of search effort that is freely chosen can therefore never be Pareto improving for a naive agent 14

Referring to Akerlof (1991)'s view on procrastination, O'Donoghue and Rabin (1999) argue, however, that the aforementioned welfare criterion is too strong for a welfare analysis. The reason is that preferences of the current self are biased in that he faces a self-control problem and the naive agent, in addition, a misperception problem (Gruber and Köszegi, 2000, 2001). The sophisticated future self, by contrast, does not face these problems. It is therefore argued that the preferences of a future sophisticated self are more appropriate to base welfare analysis on. In the context of time-inconsistency, Noor (2011) confirms that normative judgments cannot be based on revealed preferences but instead on what he calls normative preferences, that is, those which reflect the choices the individual thinks he should make. He concludes that normative preferences are those of the future self in our context. Following these papers, the welfare of the naive agent is represented by the same curve as the one of the sophisticated agent, i.e. by $r(\sigma \mid b)$. His level of welfare at the free choice $\sigma_{n}^{B}$ can then be read from point $\mathrm{K}$ in Figure 1. Consequently, imposing job search requirements is now welfare enhancing for the naive agent until the search requirement associated to point $\mathrm{H}$ in Figure 1 is reached. For the sophisticated agent, the same is true until point $\mathrm{H}$ in Figure 2,

Job search monitoring is not only welfare enhancing for the unemployed with hyperbolic preferences. In addition, since, by Proposition 5 , the exit rate from unemployment increases monotonically with the level of the job search requirement, society thereby reduces expenditures on UB. Since the cost of monitoring is negligible relative to the cost of UB (e.g. Boone et al., 2007; Cockx et al., 2011), this suggests that social efficiency can be attained by setting the job search requirement between $\mathrm{A}$

\footnotetext{
${ }^{14} \mathrm{~A}$ similar conclusion applies for an exponential agent.
} 
and $\mathrm{H}$ in Figures 1 and 2 In that range, a further rise in the search requirement entails a trade-off as on the one hand the rise in exit rates from unemployment reduces expenditures on UB and on the other hand the long-term welfare of the impatient UB claimants shrinks.

Yet, because the unemployed may decide not to comply with the job search requirement, social efficiency may not be attainable. In general, we cannot be sure that non-compliance is an issue, because we cannot in general exclude that an UB claimant would only decide to stop complying if the requirement is set beyond point $\mathrm{H}$ (Proposition 2 (iii)). We can only state that this is more likely to be the case for naive than for sophisticated agents. However, our analysis makes clear that it is crucial to take non-compliance into account when evaluating the social efficiency of job search monitoring. It may well be that another policy, such as job search assistance, imposes less costs on the current self and therefore be socially more efficient. Moreover, until now we assumed that the government can monitor search effort perfectly. In reality, there will be imperfections, which may also affect the social efficiency of a monitoring system. In the next section we discuss the implications of imperfections in the monitoring technology.

\section{The Consequences of an Imperfect Monitoring Technology}

Up to this moment, we assumed that the monitoring technology was perfect, so that compliance could be detected without any error. This is an extreme assumption. In this section we therefore relax this assumption and allow for measurement errors. We show that in this case the likelihood of attaining social efficiency through monitoring job search is further reduced. We first define a number of possible imperfections in the monitoring technology. Subsequently, we show how this affects the optimization problem and the corresponding solution. Finally, to get more insight, we illustrate the solution graphically.

\subsection{Defining Imperfections in the Monitoring Technology}

We discuss four types of imperfections in the monitoring technology. First, we follow Abbring et al. (2005) and assume that below the threshold $\bar{\sigma}$ the probability of a sanction is strictly positive, but smaller than one:

$$
p(\sigma)=\left\{\begin{array}{cl}
p_{0} \in(0,1) & \text { for } \sigma<\bar{\sigma} \text { and } \\
0 & \text { for } \sigma \geq \bar{\sigma} .
\end{array}\right.
$$

This could reflect a monitoring scheme in which a UB claimant is not sanctioned if search effort is above the threshold $\bar{\sigma}$, but if it is not, other random factors, uncorrelated with job search effort, determine whether a sanction is imposed or not. This could be the case in a system in which caseworkers have a lot of discretion in determining who is sanctioned and/or in situations where criteria, such as social need, are taken into account. This is an instructive case, since, as discussed below, it already provides an important insight in why imperfections in the monitoring technology 
lead to more non-compliance and, therefore, makes it more difficult to attain social efficiency by raising job search requirements. Nevertheless, that search intensity below the threshold does not play any role is extreme. This is remedied in the subsequent considered imperfection.

Second, we allow for imperfect measurement of job search effort. We assume a log-linear rather than a linear measurement error as assumed by Boone et al. (2007), since we believe that this is more natural for an error on a non-negative variable. If $\sigma_{0}$ denotes the search effort observed by the caseworker and $\varepsilon$ the measurement error on the logarithm of the realized search effort:

$$
\log \sigma_{o}=\log \sigma+\varepsilon
$$

where $\varepsilon \in[-\underline{\varepsilon}, \bar{\varepsilon}]$ for $-\underline{\varepsilon}<0<\bar{\varepsilon}$, and $G(\varepsilon)$ denotes the cumulative distribution function of $\varepsilon$. The width of the support of $\varepsilon$ is inversely proportional to the precision of the monitoring technology. The policy maker can invest resources to increase this precision. Boone et al. (2007) discuss how to determine the optimal amount of resources to invest. If $\bar{\varepsilon}=\underline{\varepsilon}$, then the measurement error is symmetric around zero. We allow for asymmetry, since the policy maker can be differently concerned with type I and type II errors. Type I errors occur if individuals are sanctioned despite the actual search effort satisfies the requirement, while Type II errors occur if the individuals are not sanctioned even if they should have been on the basis of the actual effort. For Type I errors, the magnitude of $\underline{\varepsilon}$ matters, while for Type II, it is the size of $\bar{\varepsilon}$ that is relevant.

Since the evaluation of job search effort now depends on the random observed search effort, the probability $p(\bar{\sigma} / \sigma)$ of a sanction,

$$
p\left(\frac{\bar{\sigma}}{\sigma}\right) \equiv \operatorname{Prob}\left(\sigma_{o}<\bar{\sigma}\right)=\operatorname{Prob}\left(\log \sigma_{o}<\log \bar{\sigma}\right)=\operatorname{Prob}\left[\varepsilon<\log \left(\frac{\bar{\sigma}}{\sigma}\right)\right]=G\left[\log \left(\frac{\bar{\sigma}}{\sigma}\right)\right],
$$

clearly declines with $\sigma$,

$$
\frac{\partial p\left(\frac{\bar{\sigma}}{\sigma}\right)}{\partial \sigma}=-\frac{g\left[\log \left(\frac{\bar{\sigma}}{\sigma}\right)\right]}{\sigma}<0
$$

where $g($.$) denotes the density function of \varepsilon$. So, in contrast to the first mentioned imperfect technology, measurement error implies that the sanction probability strictly decreases with search effort.

If we assume, e.g., that $\varepsilon$ is uniformly distributed over its support, we obtain 15

$$
p\left(\frac{\bar{\sigma}}{\sigma}\right) \equiv\left\{\begin{array}{cl}
1 & \text { for } \sigma \leq \bar{\sigma} \exp (-\bar{\varepsilon}) \\
\int_{-\underline{\varepsilon}}^{\log \left(\frac{\bar{\sigma}}{\sigma}\right)} g(\varepsilon) d \varepsilon=\frac{1}{\underline{\varepsilon}+\bar{\varepsilon}}\left[\log \left(\frac{\bar{\sigma}}{\sigma}\right)+\underline{\varepsilon}\right] & \text { for } \bar{\sigma} \exp (-\bar{\varepsilon})<\sigma<\bar{\sigma} \exp (\underline{\varepsilon}) \\
0 & \text { for } \sigma \geq \bar{\sigma}_{p} \equiv \bar{\sigma} \exp (\underline{\varepsilon})
\end{array}\right.
$$

As (21) shows, the level of actual search effort $\sigma$ needed to reach a certain sanction probability lies above the level required in the absence of measurement error $\varepsilon$. In particular, to avoid a sanction for sure the actual search effort level is $\bar{\sigma}_{p} \equiv \bar{\sigma} \exp (\underline{\varepsilon})>\bar{\sigma}$. This equation also shows that by the

\footnotetext{
${ }^{15}$ We use that $\log (\bar{\sigma} / \sigma) \geq \bar{\varepsilon} \Leftrightarrow \sigma \leq \bar{\sigma} \exp (-\bar{\varepsilon})$ and that $\log (\bar{\sigma} / \sigma) \leq-\underline{\varepsilon} \Leftrightarrow \sigma \geq \bar{\sigma} \exp (\underline{\varepsilon})$.
} 
introduction of a measurement error a third regime emerges, aside compliance and non-compliance. In this regime the observed search effort may or may not be above the search requirement $\bar{\sigma}$, depending on the level of search effort and the size of the measurement error. We label this regime as one of partial compliance.

To avoid a cumbersome description of the solutions in all three regimes and because the regime of non-compliance for sure (i.e. $\sigma \leq \bar{\sigma} \exp (-\bar{\varepsilon})$ ) is less interesting, given the focus of this paper, we will only consider below the case in which $\bar{\varepsilon} \rightarrow+\infty$, so that $\bar{\sigma} \exp (-\bar{\varepsilon})=0$ and non-compliance for sure never occurs. The full compliance regime becomes irrelevant if $\underline{\varepsilon} \rightarrow+\infty$, so that $\sigma_{p}=$ $\bar{\sigma} \exp (\underline{\varepsilon}) \rightarrow+\infty$. This limit case means that the unemployed is never sure that his search effort is high enough. It amounts to considering that part of his effort is impossible to observe. This can be the case if search effort is realized through informal channels that by the definition the case worker cannot observe van den Berg and van der Klaauw (2006). We will discuss this case in the analysis below.

A third type of monitoring imperfection occurs if, aside from the measurement error in realized search effort, the search requirement is not sharply defined and may depend on the appreciation of caseworkers. Cockx et al. (2011) consider this case. A consequence is that not only the observed search effort is random, but also the search requirement $\bar{\sigma}$. It is not difficult to see that one can then define a new random variable $\tilde{\varepsilon} \equiv \bar{\sigma} / \exp (\varepsilon)$ with support $[\underline{\tilde{\varepsilon}}, \overline{\tilde{\varepsilon}}]$ and that the probability of a sanction becomes:

$$
\operatorname{Prob}\left(\sigma_{o}<\bar{\sigma}\right)=\operatorname{Prob}(\sigma \exp (\varepsilon)<\bar{\sigma})=\operatorname{Prob}(\tilde{\varepsilon}>\sigma)=1-\tilde{G}(\sigma)=\tilde{p}(\sigma)
$$

where $\tilde{G}(\tilde{\sigma})$ denotes the cumulative distribution function of $\tilde{\sigma}$. It follows that $\tilde{p}^{\prime}(\sigma)=-\tilde{g}(\sigma)<0$.

The consequences of this additional monitoring imperfection are thus not very important. The sanction probability does no longer directly depend on the formal search requirement $\bar{\sigma}$, but this does not fundamentally affect the nature of the monitoring technology, since the actual search requirement at which the sanction probability falls to zero is given by $\overline{\tilde{\varepsilon}}$ instead of by $\bar{\sigma}_{p}$. We therefore leave an analysis of this case to the reader.

Finally, up to this point we assumed that job search effort is monitored at the end of each period. In reality, monitoring occurs at regular predetermined moments in time. This means that a defier will be detected less rapidly, than in case of a continuous evaluation. Hence, this makes it more likely that prior to the period in which the evaluation takes place benefit claimants stops complying and hence strengthens our case that imperfections in the monitoring technology make it less likely that job search monitoring can unambiguously raise social welfare. For impatient agents this issue is even more important than for exponential agents, since they will discount the future more and therefore underestimate the cost of non-compliance. However, we will not model this imperfection, since this requires modeling non-stationary behavior, which complicates the model dramatically (see e.g. Cockx et al., 2011). An alternative would be to assume that the monitoring 
occurs at random instants in time (see e.g . Boone et al., 2007). In this case the model remains stationary, but, since it cannot provide us with more insights than those that we just provided in words, we do not develop such a model in this paper.

\subsection{The Optimization Problem, the Solution and Welfare Implications}

We now formulate the optimization problem of a benefit claimant whose job search effort is monitored with measurement error, but for whom the requirement $\bar{\sigma}$ is sharply defined. As mentioned, we ignore the regime of perfect non-compliance by assuming that $\bar{\varepsilon} \rightarrow \infty$, so that the agent faces at most the choice between perfect and partial compliance. For $j \in\{s, n, e\}$ :

$$
\begin{aligned}
\tilde{\Omega}_{j p}^{c}= & \max _{j}\left\{B_{j}^{c}(\bar{\sigma}), P_{j}^{c}\right\} \\
B_{j}^{c}\left(\bar{\sigma}_{p}\right)= & \max _{\sigma \geq \bar{\sigma}_{p}} W\left(\sigma, B_{j}^{f}(\bar{\sigma}) \mid b, \beta \delta\right) \\
P_{j}^{c}(\bar{\sigma})= & \max _{\sigma} W_{p}\left(\sigma, P_{j}^{f}(\bar{\sigma}) \mid b, \beta \delta\right) \\
W_{p}\left(\sigma, P_{j}^{f}(\bar{\sigma}) \mid b, \beta \delta\right) \equiv & u(b)-c(\sigma)+\beta \delta\left\{\lambda(\sigma) E_{F}\left[\max \left(V_{j}^{f}(w), p\left(\frac{\bar{\sigma}}{\sigma}\right) Z_{j}^{f}+\left[1-p\left(\frac{\bar{\sigma}}{\sigma}\right)\right] P_{j}^{f}(\bar{\sigma})\right)\right]\right. \\
& \left.+(1-\lambda(\sigma))\left[p\left(\frac{\bar{\sigma}}{\sigma}\right) Z_{j}^{f}+\left[1-p\left(\frac{\bar{\sigma}}{\sigma}\right)\right] P_{j}^{f}(\bar{\sigma})\right]\right\}
\end{aligned}
$$

where, as before, $B_{j}^{c}\left(\bar{\sigma}_{p}\right)$ is the expected lifetime utility of the perfectly complying current self (2) and $P_{j}^{c}(\bar{\sigma})$ of the partial complier. With superscript $f$ instead of $c$ these refer to the utility levels of the future selves. $B_{j}^{c}\left(\bar{\sigma}_{p}\right)$ depends on $\bar{\sigma}_{p}$ and not on $\bar{\sigma}$ as a consequence of the measurement error. $P_{j}^{c}(\bar{\sigma})$ depends on $\bar{\sigma}$, since the sanction probability $p(\bar{\sigma} / \sigma)$ is a function of it. In case of partial compliance, unless the benefit claimant has found a job, he is sanctioned at the end of each period with probability $p(\bar{\sigma} / \sigma)$, in which case lifetime utility of the future self drops to $Z_{j}^{f}$. With probability $[1-p(\bar{\sigma} / \sigma)]$ he is not sanctioned and continues to comply partially.

Following the same arguments as for the case of perfect monitoring, in case of perfect compliance, the interior solution verifies the implicit equations (14) and 15 for $U=B$. For the partial complier, the corresponding equations are :

$$
\begin{aligned}
R_{p}(\sigma, x \mid b, \bar{\sigma}) & \equiv u(b)+\frac{\delta \lambda(\sigma)}{1-\delta(1-q)} Q(x)-c(\sigma)-u(x)-\frac{\delta p(\bar{\sigma} / \sigma)}{(1-\delta)}\left[u(x)-u\left(x_{j}^{Z}\right)\right]=0, \\
S_{p}(\sigma, x \mid \beta, \bar{\sigma}) & \equiv \frac{\beta \delta \lambda^{\prime}(\sigma)}{1-\delta(1-q)} Q(x)+\frac{\beta \delta}{1-\delta} \frac{\partial p(\bar{\sigma} / \sigma)}{\partial \sigma}\left[u\left(x_{j}^{Z}\right)-u(x)\right]-c^{\prime}(\sigma)=0
\end{aligned}
$$

where $x_{j}^{Z}$ is the optimal reservation wage for a sanctioned individual. Note that the curves defined by the two above implicit equations shift when the level of observed search requirement $\bar{\sigma}$, which is present in the sanction probability $p(\bar{\sigma} / \sigma)$, changes. In case of partial compliance, continuing search is more costly than in case of perfect compliance, since the benefit claimant risks to be sanctioned. This explains the additional negative term $-[\delta p(\bar{\sigma} / \sigma) /(1-\delta)]\left[u(x)-u\left(x_{j}^{Z}\right)\right]$ in $(27)$. On the other hand, the marginal benefit of search effort increases for the partial complier, since he can reduce 
the probability of being sanctioned by increasing search effort. This explains the additional positive term $[\beta \delta /(1-\delta)][\partial p(\bar{\sigma} / \sigma) / \partial \sigma]\left[u\left(x_{j}^{Z}\right)-u(x)\right]$ in 28 .

Before discussing the implications of these additional terms on the solution, we first consider the simpler case in which search effort is perfectly measured, but in which the sanction probability is below one. This case is defined by Equation (17). Since in this case $\partial p(\bar{\sigma} / \sigma) / \partial \sigma=0$, the first order condition of search effort is the same for partial and perfect compliance. The first order condition of the reservation wage is given by (27) in which $p(\bar{\sigma} / \sigma)$ is replaced by $p_{0}$. If in the limit $p_{0}=0$ this corresponds to the condition in case of perfect compliance (14) for $\mathrm{U}=\mathrm{B})$. If in the limit $p_{0}=1$ the reservation wage is still higher than in case of perfect non-compliance ( $\sqrt{14})$ for $U=Z$ ), because the expected instantaneous income is higher: $b>z$. Because the sanction probability, $p_{0}>0$, does not depend on $\sigma$, the curve describing the reservation wage (and hence the expected utility), lies below the corresponding curve in case of perfect compliance and above the one under perfect non-compliance. Consequently, since the lifetime utility of the current self is ordered in the same way as that of the future self, the benefit claimant will stop complying at lower levels of search intensity than in a perfect monitoring scheme.

For the more complicated case 19 , this reasoning holds as well, but we need to take into account that the sanction probability now varies with $\sigma$ and $\bar{\sigma}$. Denote $x=r_{p}(\sigma \mid b, \bar{\sigma})$ the explicit function corresponding to the implicit relationship $R_{p}(\sigma, x \mid b, \bar{\sigma})=0$. When $\sigma$ reaches the actual search requirement level $\bar{\sigma}_{p}$ defined by (21), the sanction probability becomes zero and therefore $r(\sigma \mid b)=r_{p}(\sigma \mid b, \bar{\sigma}) \forall \sigma \geq \bar{\sigma}_{p}$. Since $\partial p(\bar{\sigma} / \sigma) / \partial \sigma<0$, the sanction probability is strictly larger than zero for any $\sigma<\bar{\sigma}_{p}$ and by assumption strictly smaller than one for any $\sigma>0$. By a similar reasoning as in the previous paragraph, this means that $r(\sigma \mid b)>r_{p}(\sigma \mid b, \bar{\sigma})>r(\sigma \mid z)$ for any $\sigma<\bar{\sigma}_{p}$. Hence, the UB claimant will here also stop complying at a lower level of search effort than in the absence of measurement error.

Sometimes perfect compliance may not even be feasible. For instance, if, as commonly assumed, the lower bound of the support of the measurement error tends to minus infinity $(\underline{\varepsilon} \rightarrow+\infty)$, the sanction probability can never be zero and partial compliance is the best that can be achieved. This does not mean one could not achieve Pareto improvements for the future selves of procrastinating UB claimants (and for the current selves if these claimants are sophisticated). For, as reflected by the additional positive term in the first order condition for $\sigma$ given by (28), a partially complying unemployed worker also has an incentive to search more intensively for jobs than in the absence of a monitoring scheme. However, as shown in the previous paragraph, for any given search effort $\sigma$, the expected lifetime utility of the partial complier is always strictly lower than in the case of perfect compliance. This means that the Pareto efficient region of a perfect complier cannot be attained. Consequently, if whatever the search effort level there still is a non zero risk of being sanctioned, social efficiency cannot be achieved by an imperfect job search monitoring technology. 


\subsection{Graphical Analysis of a Monitoring Scheme with Measurement Error}

In order to illustrate the analysis in the previous paragraph, we propose, as for the case of perfect monitoring, a graphical representation of the solution. The first-order condition with respect to search effort (28) describes a locus that lies above the one in the perfect complier case in a space where realized search effort $\sigma$ is on the horizontal axis and $x$ on the vertical one. In Proposition 6 (i) we show that the implicit function $R_{p}(\sigma, x \mid b, \bar{\sigma})=0$ is, as for the perfect complier, first increasing in the $(\sigma, x)$ plane, attaining a maximum at the optimal choice for a partially complying exponential agent facing a job search requirement equal to $\bar{\sigma}$, i.e. at $\sigma_{e}^{P}(\bar{\sigma})$, and subsequently monotonically decreasing. The implicit function $S_{p}(\sigma, x \mid \beta, \bar{\sigma})=0$ is no longer generally a strictly decreasing function in the $(\sigma, x)$ plane. For particular distributions of the measurement error it might be increasing or non-monotonic and, consequently, the solution may no longer be unique (cf. Proposition 3). However, in Proposition 6 (ii) we provide sufficient conditions for this function to be strictly decreasing and therefore for uniqueness: the relationship $\sigma \mapsto p(\bar{\sigma} / \sigma)$ should not be too sensitive to changes in $\sigma$ and be convex. Moreover, it is shown that, if these sufficient conditions are satisfied, $S_{p}(\sigma, x \mid \beta, \bar{\sigma})=0$ describes a curve in the $(\sigma, x)$ plane that declines more steeply than in the case of perfect compliance. In the graphical analysis we assume that these sufficient conditions are satisfied.

\section{Proposition 6.}

Let $x=r_{p}(\sigma \mid b, \bar{\sigma})$ and $x=s_{p}(\sigma \mid \beta, \bar{\sigma})$ denote the explicit functions corresponding to the implicit functions $R_{p}(\sigma, x \mid b, \bar{\sigma})=0$ and $S_{p}(\sigma, x \mid \beta, \bar{\sigma})=0$. Then $\forall \beta \in(0,1], \forall b \in \mathbb{R}^{+}$and

(i) $\forall \sigma \lesseqgtr \sigma_{e}^{P}(\bar{\sigma}): \frac{\partial r_{p}(\sigma \mid b, \bar{\sigma})}{\partial \sigma}=S_{p}\left[\sigma, r_{p}(\sigma \mid b, \bar{\sigma}) \mid 1, \bar{\sigma}\right]\left[u^{\prime}\left(r_{p}(\sigma \mid b, \bar{\sigma})\right)\left(1+\frac{\delta h\left[\sigma, r_{p}(\sigma \mid b, \bar{\sigma})\right]}{1-\delta(1-q)}+\frac{\delta p\left(\frac{\bar{\sigma}}{\sigma}\right)}{1-\delta}\right)\right]^{-1} \gtreqless 0$, where $S_{p}\left[\sigma_{e}^{P}(\bar{\sigma}), r_{p}\left(\sigma_{e}^{P}(\bar{\sigma}) \mid b, \bar{\sigma}\right) \mid 1, \bar{\sigma}\right]=0$,

(ii) $\forall \sigma \in \mathbb{R}^{+}:$if $\frac{\partial^{2} p\left(\frac{\bar{\sigma}}{\sigma}\right)}{\partial \sigma^{2}} \geq q^{16}$ then $\frac{\partial s_{p}(\sigma \mid \beta, \bar{\sigma})}{\partial \sigma} \lessgtr 0$ iff $\lambda^{\prime}(\sigma) \bar{F}\left[s_{p}(\sigma \mid \beta, \bar{\sigma})\right](1-\delta) \gtrless-\frac{\partial p\left(\frac{\bar{\sigma}}{\sigma}\right)}{\partial \sigma}[1-\delta(1-q)]$; If $\frac{\partial s_{p}(\sigma \mid \beta, \bar{\sigma})}{\partial \sigma}<0$ then $\frac{\partial s_{p}(\sigma \mid \beta, \bar{\sigma})}{\partial \sigma}<\frac{\partial s(\sigma \mid \beta)}{\partial \sigma}$,

(iii) $\forall \sigma \in \mathbb{R}^{+}: \frac{\partial r_{p}(\sigma \mid b, \bar{\sigma})}{\partial b}>0, \frac{\partial r_{p}(\sigma \mid b, \bar{\sigma})}{\partial \beta}=0$ and $\frac{\partial r_{p}(\sigma \mid b, \bar{\sigma})}{\partial \bar{\sigma}}<0$,

(iv) $\forall \sigma \in \mathbb{R}^{+}: \frac{\partial s_{p}(\sigma \mid \beta, \bar{\sigma})}{\partial b}=0, \frac{\partial s_{p}(\sigma \mid \beta, \bar{\sigma})}{\partial \beta}>0$ and $\frac{\partial s_{p}(\sigma \mid \beta, \bar{\sigma})}{\partial \bar{\sigma}}>0$.

Proof. See Appendix F

In Figure 3, we illustrate the solution for a sophisticated agent and leave it to the reader to construct the corresponding figure for the naive agent. Denote $\bar{\sigma}_{p s *}^{\max }$ the requirement on observed search effort such that the sophisticated UB claimant is indifferent between complying perfectly and only partially. The curves are drawn for this specific value of the requirement. Denote also

\footnotetext{
${ }^{16}$ This is satisfied if $\varepsilon$ is uniformly distributed over its support.
} 
$\bar{\sigma}_{p s}^{\max }$ the realized search level corresponding to $\bar{\sigma}_{p s *}^{\max }[1]$ We now explain how the threshold is determined. The curve through the points $\mathrm{Q}$ and $\mathrm{N}$ represents $r_{p}\left(\sigma \mid b, \bar{\sigma}_{p s *}^{\max }\right)$. It designates the level of the reservation wage for any given $\sigma$ in case of partial compliance given the imposed job search requirement $\bar{\sigma}_{p s *}^{\max }$. Since $s_{p}\left(\sigma \mid \beta=1, \bar{\sigma}_{p s *}^{\max }\right)$ is steeper than $s(\sigma \mid \beta=1)$, the maximum of $r_{p}\left(\sigma \mid b, \bar{\sigma}_{p s *}^{\max }\right)$ is attained at point $\mathrm{N}$ to the right of point $\mathrm{A}$, the maximum of an exponential agent's free choice in the absence of monitoring.

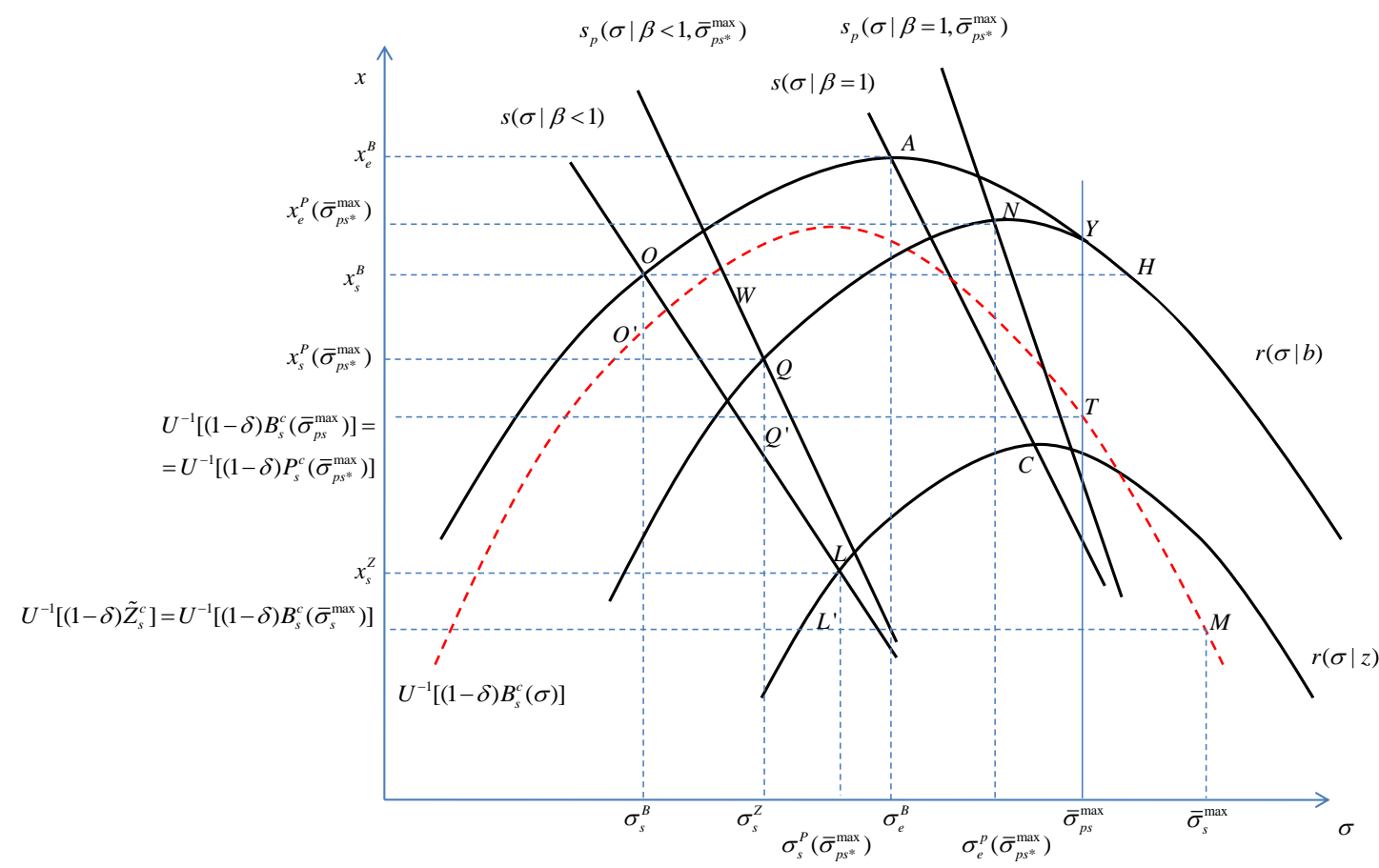

Figure 3: The Solution for the Sophisticated Agent in Case of Imperfect Monitoring with Imposed Search Requirement Set Equal to $\bar{\sigma}_{p s *}^{\max }$, the Level at which the Agent Is Indifferent Between Perfect and Partial Compliance. $x=$ reservation wage; $\sigma=$ realized search effort

The optimal solution for the partially complying sophisticated agent can be found at the intersection between $r_{p}\left(\sigma \mid b, \bar{\sigma}_{p s *}^{\max }\right)$ and $s_{p}\left(\sigma \mid \beta<1, \bar{\sigma}_{p s *}^{\max }\right)$ at point $\mathrm{Q}$. As in the case of the exponential agent, the search effort of the partial complier is higher than the level chosen by the sophisticated agent in the absence of monitoring (at point $\mathrm{O}$ ). The corresponding (function of the) lifetime utility of the current self is lower at point Q'. If the utility of the perfectly complying current self drops below this level, i.e. if the actual search requirement becomes higher than $\bar{\sigma}_{p s}^{\max }$ (point $\mathrm{T}$ on the

\footnotetext{
${ }^{17}$ There is a one to one relationship between these two values. Since the sanction probability is nil if observed search effort lies above the threshold $\bar{\sigma}_{p s *}^{\max }$, by $\left[21\right.$, is not difficult to see that $\bar{\sigma}_{p s *}^{\max }=\bar{\sigma}_{p s}^{\max } \exp (-\underline{\varepsilon})$.
} 
interrupted curve), the UB claimant will shift to the regime of partial compliance. So, $\bar{\sigma}_{p s *}^{\max }$ verifies $B_{s}^{c}\left(\bar{\sigma}_{p s *}^{\max }\right)=P_{s}^{c}\left(\bar{\sigma}_{p s *}^{\max }\right)$. At this maximum level of the actual job search requirement, the sanction probability is zero. From the figure one can also easily see that the sophisticated agent stops complying at a lower search effort than in the case of a perfect monitoring scheme: $\bar{\sigma}_{p s}^{\max }<\bar{\sigma}_{s}^{\max }$ (point $\mathrm{M}$ is to the right of point $\mathrm{T})$.

In Figure 3 one can also clearly see that part (from $\mathrm{Y}$ to $\mathrm{H}$ ) of the socially efficient region (from A to $\mathrm{H}$ ) cannot be reached. This is no general result, however. Depending on the parameters of the model, the full range $\mathrm{A}$ to $\mathrm{H}$ may be feasible or no point at all. It is clear, however that the likelihood of attaining social efficiency is reduced in case of an imperfect monitoring scheme induced by measurement error of search effort. If, as already mentioned, the lower bound of the support of the measurement error tends to minus infinity, then social efficiency (the curve between $\mathrm{A}$ and $\mathrm{H}$ ) can never be attained, since agents can then at most partially comply with the search requirement.

\section{Conclusion}

Given the size of the insured unemployed population and the financial pressure on public budgets, many governments have intensified monitoring of job search effort of unemployed benefit recipients. If unemployed workers are exponential discounters this intensification does not unambiguously enhance social welfare, since, even if it enhances the exit rate from unemployment and thereby reduces outlays on benefit payments, it hurts the unemployed, both by the higher effort level that this monitoring imposes and by the lower expected quality jobs that are subsequently accepted as a consequence of lower reservation wages. However, if the unemployed are hyperbolic discounters, PDV and Paserman (2008) have shown that monitoring job search of the unemployed may even be Pareto improving, at least if the unemployed are sophisticated hyperbolic discounters who realize that they exert too little search effort if they are not monitored. In this research we have first confirmed this conclusion, based on a graphical analysis, and generalized it by showing that it also applies for a wider class of wage offer distributions than the aforementioned researchers considered as well as for risk averse (rather than risk neutral) individuals. In addition, we have argued that, even if the utility of the the current self does not increase in case that the unemployed are naive hyperbolic discounters, social welfare may unambiguously increase, if, as argued in the literature, the welfare of the unemployed is evaluated according to a social preference function that disregards the biases induced by self-control and misperception problems.

These conclusions with regards to the optimality of a monitoring scheme for hyperbolic discounting unemployed benefit recipients are, however, inconsistent with empirical studies that report ambiguous effects of monitoring of job search effort on the job finding rate and on job quality and that finds that monitoring may sometimes induce exits out of the labor force and, hence, lead to lower welfare levels for the unemployed. We have argued in this paper that this mixed evidence 
can nevertheless be compatible with the hypothesis that the unemployed procrastinate. The reason is that the aforementioned optimality results are derived under two strong and unrealistic assumptions. First, the unemployed can only exit towards employment. In reality, they can in particular withdraw from the claimant register and live from welfare benefits, solidarity of family members or charities. Taking this alternative into account, and assuming that job search effort is then no more monitored, we have shown that social efficiency may no more be attainable. Increasing job search requirements to a level that is optimal from the perspective of the future selves or from the perspective of society may eventually lead to a sub-optimal level of search effort and a long-run utility that is even lower than in the absence of job search requirements. A second oversimplifying assumption is that monitoring is perfect, that is: search effort is observed without error and can be raised up to a desired level by the UI agency. When monitoring becomes imperfect, the likelihood that monitoring of search effort increases welfare shrinks. In particular, if whatever the search effort level there still is a non zero risk of being sanctioned, our analysis demonstrates that social efficiency can then not be achieved. Consequently, even if the unemployed are hyperbolic discounters, other policy instruments, such as counseling, could improve on monitoring schemes from a social welfare point of view. However, whether this is indeed the case remains matter for future empirical research.

\section{Acknowledgements}

We thank Mathias Hungerbühler for his valuable comments and discussions. We are also very grateful for the comments received from the participants of the seminars at CORE (IUAP/PAI Public economics day), KULeuven (Day for Labour Economists), Ghent University (internal seminar), Louvain-la-Neuve (Doctoral Workshops) and the EALE annual conference in Bonn. Corinna Ghirelli acknowledges financial support of the Special Research Fund ("BOF") of Ghent University as well as of the National Bank of Belgium (project code 05Y00110). The usual disclaimer applies.

\section{References}

Abbring, J. H., G. J. Berg, and J. C. Ours (2005). The Effect of Unemployment Insurance Sanctions on the Transition Rate from Unemployment to Employment. Economic Journal 115(505), 602630 .

Ainslie, G. (1992). Picoeconomics: The Strategic Interaction of Successive Motivational States within the Person. Cambridge University Press.

Akerlof, G. A. (1991). Procrastination and Obedience. American Economic Review 81(2), 1-19. 
Angeletos, G., D. Laibson, A. Repetto, J. Tobacman, and S. Weinberg (2001). The Hyperbolic Consumption Model: Calibration, Simulation, and Empirical Evaluation. The Journal of Economic Perspectives 15(3), 47-68.

Ashenfelter, O., D. Ashmore, and O. Deschenes (2005). Do unemployment insurance recipients actively seek work? Evidence from randomized trials in four U.S. states. Journal of Econometrics $125(1-2), 53-75$.

Augenblick, N., M. Niederle, and C. Sprenger (2013). Working Over Time: Dynamic Inconsistency in Real Effort Tasks. Working Paper 18734, NBER.

Boone, J., P. Fredriksson, B. Holmlund, and J. C. van Ours (2007). Optimal Unemployment Insurance with Monitoring and Sanctions. Economic Journal 117(518), 399-421.

Boone, J. and J. C. van Ours (2006). Modeling Financial Incentives to Get the Unemployed Back to Work. Journal of Institutional and Theoretical Economics 162(2), 227-252.

Carrillo, J. D. and T. Mariotti (2000). Strategic Ignorance as a Self-Disciplining Device. The Review of Economic Studies 67(3), 529-544.

Cockx, B. and M. Dejemeppe (2012). Monitoring job search effort: An evaluation based on a regression discontinuity design. Labour Economics 19(5), 729 - 737.

Cockx, B., M. Dejemeppe, A. Launov, and B. Van der Linden (2011). Monitoring, Sanctions and Front-Loading of Job Search in a Non-Stationary Model. Working Paper 6181, IZA.

DellaVigna, S. and U. Malmendier (2006). Paying Not to Go to the Gym. American Economic Review 96(3), 694-719.

DellaVigna, S. and M. D. Paserman (2005). Job Search and Impatience. Journal of Labor Economics $23(3), 527-588$.

Engström, P., P. Hesselius, and B. Holmlund (2012). Vacancy Referrals, Job Search, and the Duration of Unemployment: A Randomized Experiment. Labour 26(4), 419-435.

Fang, H. and D. Silverman (2009). Time-Inconsistency and Welfare Program Participation: Evidence from the NLSY. International Economic Review 50(4), 1043-1077.

Fang, H. and Y. Wang (2010). Estimating Dynamic Discrete Choice Models with Hyperbolic Discounting, with an Application to Mammography Decisions. Working Paper 16438, NBER.

Frederick, S., G. Loewenstein, and T. O'Donoghue (2002). Time Discounting and Time Preference: A Critical Review. Journal of Economic Literature 40(2), 351-401. 
Goldman, S. M. (1980). Consistent Plans. The Review of Economic Studies 47(3), 533-537.

Gruber, J. and B. Köszegi (2000). Is Addiction "Rational"? Theory and Evidence. Working Paper 7507, NBER.

Gruber, J. and B. Köszegi (2001). Is Addiction "Rational"? Theory and Evidence. The Quarterly Journal of Economics 116(4), 1261-1303.

Halima, B. B. and M. A. B. Halima (2009). Time Preferences and Job Search: Evidence from France. Labour 23(3), 535-558.

Klepinger, D. H. (1998). Evaluation of the Maryland Unemployment Insurance Work Search Demonstration: Final Report. [Washington, DC]: U.S. Dept. of Labor, Employment and Training Administration, Unemployment Insurance Service.

Klepinger, D. H., T. R. Johnson, and J. M. Joesch (2002). Effects of Unemployment Insurance WorkSearch Requirements: The Maryland Experiment. Industrial and Labor Relations Review 56(1), $3-22$.

Krueger, A. and A. Mueller (2010). Job search and unemployment insurance: New evidence from time use data. Journal of Public Economics 94(3-4), 298-307.

Laibson, D. (1997). Golden Eggs and Hyperbolic Discounting. The Quarterly Journal of Economics 112(2), 443-77.

Lalive, R., J. C. van Ours, and J. Zweimüller (2006). How Changes in Financial Incentives Affect the Duration of Unemployment. The Review of Economic Studies 73(4), 1009-1038.

Loewenstein, G. and D. Prelec (1992). Anomalies in Intertemporal Choice: Evidence and an Interpretation. The Quarterly Journal of Economics 107(2), 573-97.

Loewenstein, G. and R. H. Thaler (1989). Anomalies: Intertemporal Choice. The Journal of Economic Perspectives 3(4), 181-193.

Manning, A. (2009). You can't always get what you want: The impact of the UK jobseeker's allowance. Labour Economics 16(3), 239-250.

Manning, A. (2011). Imperfect Competition in the Labour Market. In O. Ashenfelter and D. Card (Eds.), Handbook of Labor Economics, Volume 4B, Chapter 11, pp. 973-1041. North-Holland.

McVicar, D. (2008). Job search monitoring intensity, unemployment exit and job entry: Quasiexperimental evidence from the UK. Labour Economics 15(6), 1451-1468. 
Mortensen, D. T. (1986). Job search and labor market analysis. In O. Ashenfelter and R. Layard (Eds.), Handbook of Labor Economics, Volume 2 of Handbook of Labor Economics, Chapter 15, pp. 849-919. Elsevier.

Mullainathan, S. and J. Gruber (2005). Do Cigarette Taxes Make Smokers Happier? The B.E. Journal of Economic Analysis and Policy 5(1), 4.

Noor, J. (2009). Hyperbolic discounting and the standard model: Eliciting discount functions. Journal of Economic Theory 144(5), 2077-2083.

Noor, J. (2011). Temptation and Revealed Preference. Econometrica 79(2), 601-644.

O’Donoghue, T. and M. Rabin (1999). Doing It Now or Later. American Economic Review 89(1), $103-124$.

OECD (2007). Employment Outlook. Paris: OECD.

OECD (2011). Labour Force Statistics: 1989-2009. Paris: OECD.

Paserman, M. D. (2008). Job search and hyperbolic discounting: Structural estimation and policy evaluation. The Economic Journal 118, 1418-1452.

Petrongolo, B. (2009). The long-term effects of job search requirements: Evidence from the UK JSA reform. Journal of Public Economics 93(11-12), 1234-1253.

Phelps, E. S. and R. A. Pollak (1968). On Second-Best National Saving and Game-Equilibrium Growth. The Review of Economic Studies 35(2), 185-199.

Pollak, R. A. (1968). Consistent Planning. The Review of Economic Studies 35(2), 201-208.

Schelling, T. C. (1984). Self-Command in Practice, in Policy, and in a Theory of Rational Choice. The American Economic Review 74(2), 1-11.

Strotz, R. H. (1955). Myopia and Inconsistency in Dynamic Utility Maximization. The Review of Economic Studies 23(3), 165-180.

Thaler, R. H. and H. M. Shefrin (1981). An Economic Theory of Self-Control. Journal of Political Economy 89(2), 392-406.

van den Berg, G. J. (1994). The Effects of Changes of the Job Offer Arrival Rate on the Duration of Unemployment. Journal of Labor Economics 12(3), 478-98.

van den Berg, G. J. and B. van der Klaauw (2006). Counseling And Monitoring Of Unemployed Workers: Theory And Evidence From A Controlled Social Experiment. International Economic Review 47(3), 895-936. 


\section{APPENDIX}

\section{A Derivation of $W\left(\sigma, U_{j}^{f} \mid y^{U}, \beta \delta\right)$ in Equation (10)}

Proof. From (4) we can write:

$$
W\left(\sigma, U_{j}^{f} \mid y^{U}, \beta \delta\right)=u\left(y^{U}\right)-c(\sigma)+\beta \delta\left\{\lambda(\sigma) E_{F}\left\{\max \left(V(w), U^{f}\right)\right\}+(1-\lambda(\sigma)) U^{f}\right\}
$$

Using the definition of the reservation wage $x_{j}^{U}$, the expectation term in A.1 can be expanded to give:

$W\left(\sigma, U_{j}^{f} \mid y^{U}, \beta \delta\right)=u\left(y^{U}\right)-c(\sigma)+\beta \delta\left\{\lambda(\sigma)\left[\int_{\underline{w}}^{x_{j}^{U}} U_{j}^{f} d F(w)+\int_{x_{j}^{U}}^{\bar{w}} V(w) d F(w)\right]+(1-\lambda(\sigma)) U_{j}^{f}\right\}$

From (5), we obtain $V(w)=\left[u(w)+q \delta U_{j}^{f}\right] /[1-\delta(1-q)]$, which we substitute in the previous equation to obtain:

$$
\begin{aligned}
W\left(\sigma, U_{j}^{f} \mid y^{U}, \beta \delta\right) & =u\left(y^{U}\right)-c(\sigma)+\beta \delta\left\{\lambda(\sigma)\left[\int_{x_{j}^{U}}^{\bar{w}} \frac{u(w)+q \delta U_{j}^{f}}{1-\delta(1-q)}-U_{j}^{f} d F(w)\right]+U_{j}^{f}\right\} \\
& =u\left(y^{U}\right)-c(\sigma)+\beta \delta\left\{\lambda(\sigma) \int_{x_{j}^{U}}^{\bar{w}} \frac{u(w)-(1-\delta) U_{j}^{f}}{1-\delta(1-q)} d F(w)+U_{j}^{f}\right\}
\end{aligned}
$$

Plugging the definition of reservation wage (9) into (A.2) yields

$$
W\left(\sigma, U_{j}^{f} \mid y^{U}, \beta \delta\right)=u\left(y^{U}\right)-c(\sigma)+\beta \delta\left\{\lambda(\sigma) \int_{x_{j}^{U}}^{\bar{w}} \frac{\left(u(w)-u\left(x_{j}^{U}\right)\right)}{1-\delta(1-q)} d F(w)+U_{j}^{f}\right\}
$$

Finally, using the definition of $Q\left(x_{j}^{U}\right)$, one gets 10

$$
W\left(\sigma, U_{j}^{f} \mid y^{U}, \beta \delta\right)=u\left(y^{U}\right)-c(\sigma)+\beta \delta\left\{\frac{\lambda(\sigma) Q\left(x_{j}^{U}\right)}{1-\delta(1-q)}+U_{j}^{f}\right\}
$$

\section{B Proof of Proposition 1}

(i) Proof.

In (15), put $c^{\prime}(\sigma)$ in the right-hand-side. Then, take the log of both sides of this rearranged condition. Next, log differentiate this equality taking into account that $x=s(\sigma \mid \beta)$. This immediately proves (i). 
(ii) Proof.

Given that $x=r\left(\sigma \mid y^{U}\right)$ and partially differentiating (14) with respect to $\sigma$ and using the definition of $S\left(\sigma, r\left(\sigma \mid y^{U}\right) \mid \beta\right)$ in (15) yields:

$$
S\left(\sigma, r\left(\sigma \mid y^{U}\right) \mid 1\right)+\left[\frac{\delta \lambda(\sigma)}{1-\delta(1-q)} Q^{\prime}\left[r\left(\sigma \mid y^{U}\right)\right]-u^{\prime}\left[r\left(\sigma \mid y^{U}\right)\right]\right] \frac{\partial r\left(\sigma \mid y^{U}\right)}{\partial \sigma}=0
$$

From (11) $Q^{\prime}\left[r\left(\sigma \mid y^{U}\right)\right]=-\bar{F}\left[r\left(\sigma \mid y^{U}\right)\right] u^{\prime}\left[r\left(\sigma \mid y^{U}\right)\right]$. Inserting this in (A.4) and rearranging gives the desired expression. Since the second product is strictly positive, $\partial r\left(\sigma \mid y^{U}\right) / \partial \sigma$ has the same sign as $S\left(\sigma, r\left(\sigma \mid y^{U}\right) \mid 1\right)$. Since this corresponds to the first order condition of search effort for an exponential agent, we have that $S\left(\sigma_{e}^{U}, r\left(\sigma \mid y^{U}\right) \mid 1\right)=0$ and positive (negative) for $\sigma<\sigma_{e}^{U}\left(\sigma>\sigma_{e}^{U}\right)$.

(iii) Proof.

Given that $x=s(\sigma \mid \beta)$, partially differentiating (15) with respect to $\beta$, and noting that $Q^{\prime}\left[r\left(\sigma \mid y^{U}\right)\right]<0$ yields:

$$
\frac{\partial s(\sigma \mid \beta)}{\partial \beta}=-\frac{Q\left[r\left(\sigma \mid y^{U}\right)\right]}{Q^{\prime}\left[r\left(\sigma \mid y^{U}\right)\right]}>0
$$

Since $s(\sigma \mid \beta)$ is independent of $y^{U}, \partial s(\sigma \mid \beta) / \partial y^{U}=0$.

(iv) Proof.

Given that $x=r\left(\sigma \mid y^{U}\right)$, partially differentiating (14) with respect to $y^{U}$, and following the same steps as in point (ii) yields:

$$
\frac{\partial r\left(\sigma \mid y^{U}\right)}{\partial y^{U}}=\frac{[1-\delta(1-q)] u^{\prime}\left(y^{U}\right)}{\left[1-\delta(1-q)+\delta h\left[\sigma, r\left(\sigma \mid y^{U}\right)\right]\right] u^{\prime}\left[r\left(\sigma \mid y^{U}\right)\right]}>0
$$

Since $r\left(\sigma \mid y^{U}\right)$ is independent of $\beta, \partial r\left(\sigma \mid y^{U}\right) / \partial \beta=0$.

\section{Proof of Proposition 2}

(i) (a) Proof.

Using the definition of $B_{j}^{c}(\bar{\sigma})$ in $(2)$, noting that $B_{j}^{f}(\bar{\sigma})$ has the same expression specialized to $\beta=1$, and noting that for the current self $\beta<1$, we obtain:

$$
B_{j}^{f}(\bar{\sigma})-B_{j}^{c}(\bar{\sigma})=(1-\beta) \delta\left\{\lambda(\bar{\sigma}) E_{F}\left\{\max \left(V_{j}^{f}(w), B_{j}^{f}(\bar{\sigma})\right)\right\}+(1-\lambda(\bar{\sigma})) B_{j}^{f}(\bar{\sigma})\right\}>0
$$

This is true whether the constraint $\sigma_{n}^{B} \geq \bar{\sigma}$ binds (formally treated just above) and in the case where it does not bind. 
(i) (b) Proof.

Using the definition of $\tilde{Z}_{j}^{c}(\bar{\sigma})$ in (3), notice that $Z_{j}^{f}(\bar{\sigma})$ has the same expression specialized to $\beta=1$ apart from the fact that the future self is sanctioned, so that his income is $z$ rather than $b$ and noting that for the current self $\beta<1$. Note also that the search effort that maximizes $\tilde{Z}_{j}^{c}$ and the one that maximizes $\tilde{Z}_{j}^{f}$ are the same. Therefore:

$$
\tilde{Z}_{j}^{f}-\tilde{Z}_{j}^{c}=u(z)-u(b)+\delta(1-\beta)\left\{\frac{\lambda\left(\sigma_{j}^{z}\right)}{1-\delta(1-q)} Q\left(x_{j}^{z}\right)+Z_{j}^{f}\right\}
$$

which leads to a condition different from the one in the paper, namely:

$$
\tilde{Z}_{j}^{f} \lesseqgtr \tilde{Z}_{j}^{c} \Leftrightarrow \beta \gtreqless 1-\frac{u(b)-u(z)}{\delta\left\{\frac{\lambda\left(\sigma_{j}^{z}\right)}{1-\delta(1-q)} Q\left(x_{j}^{z}\right)+Z_{j}^{f}\right\}}
$$

(i) (c) Proof.

The naive agent assumes that his future selves will behave like exponential agents, whereas the sophisticated agent realizes that his future selves will have the same self-control problem. This means that as long as $\bar{\sigma}<\sigma_{e}^{U}$ the naive agent believes that the job search requirement will not bind in the future and will set his reservation wage at a higher level than the sophisticated agent. Since the reservation wage and the lifetime utilities of the future selves are positively related (see (9)) and since the instantaneous utility of the naive and sophisticated agents are identical if the job search requirement binds, i.e. the instantaneous utility is $u(b)-c(\bar{\sigma})$, this means that, for $\bar{\sigma}<\sigma_{e}^{U}, U_{n}^{c}(\bar{\sigma})>U_{s}^{c}(\bar{\sigma})$. However, if $\bar{\sigma} \geq \sigma_{e}^{U}$, then the job search requirement also binds for the exponential agent. In that case both the naive and the sophisticated agents realize that the requirement will bind for the future selves, so that both future selves will also set both the search effort and the reservation wage at the same level. Consequently, $U_{n}^{c}(\bar{\sigma})=U_{s}^{c}(\bar{\sigma})$.

(ii) (a) Proof.

Using (2), (4), (9) and noting that the reservation wage of the naive agent is only affected by $\bar{\sigma}$ if $\bar{\sigma}>\sigma_{e}^{B}$, we can write:

$$
B_{n}^{c}(\bar{\sigma})=\max _{\sigma \geq \bar{\sigma}} u(b)-c(\sigma)+\beta \delta\left\{\frac{\lambda(\sigma)}{1-\delta(1-q)} Q\left[x_{e}^{B}(\bar{\sigma})\right]+\frac{u\left[x_{e}^{B}(\bar{\sigma})\right]}{1-\delta}\right\}
$$

where $x_{e}^{B}(\bar{\sigma}) \equiv 1_{\left\{\bar{\sigma} \leq \sigma_{e}^{B}\right\}} x_{e}^{B}+1_{\left\{\bar{\sigma}>\sigma_{e}^{B}\right\}} r(\bar{\sigma} \mid b)$ and $1_{\{A\}}=1$ if $A$ is true and $1_{\{A\}}=0$ otherwise. The search effort solving the maximization problem (A.8) is equal to $\sigma_{n}^{B}$ if the latter is strictly higher than $\bar{\sigma}\left(\right.$ with $x_{e}^{B}(\bar{\sigma})=x_{e}^{B}$ ) and $\bar{\sigma}$ otherwise. 
Partially differentiating $B_{n}^{c}(\bar{\sigma})$ in A.8 with respect to $\bar{\sigma}$ is equal to zero if $\sigma_{n}^{B}>\bar{\sigma}$. Otherwise, using that $Q^{\prime}\left[r\left(\sigma \mid y^{U}\right)\right]=-\bar{F}\left[r\left(\sigma \mid y^{U}\right)\right] u^{\prime}\left[r\left(\sigma \mid y^{U}\right)\right]$ :

$\frac{\partial B_{n}^{c}(\bar{\sigma})}{\partial \bar{\sigma}}=S\left(\bar{\sigma}, x_{e}^{B}(\bar{\sigma}) \mid \beta\right)+1_{\left\{\bar{\sigma}>\sigma_{e}^{B}\right\}} \beta \delta \frac{[(1-\delta)(1-h[\bar{\sigma}, r(\bar{\sigma} \mid b)]+\delta q)] u^{\prime}\left[r\left(\bar{\sigma} \mid y^{U}\right)\right]}{[1-\delta(1-q)](1-\delta)} \frac{\partial r(\bar{\sigma} \mid b)}{\partial \bar{\sigma}}$

Since $S\left(\bar{\sigma}, x_{e}^{B}(\bar{\sigma}) \mid \beta\right) \leqq 0$ iff $\bar{\sigma} \geqq \sigma_{n}^{B}$ and since, by Proposition 1 (ii) $\partial r(\bar{\sigma} \mid b) / \partial \bar{\sigma}<0$ iff $\bar{\sigma}>\sigma_{e}^{B}, \partial B_{n}^{c}(\bar{\sigma}) / \partial \bar{\sigma} \leqq 0$ iff $\bar{\sigma} \geqq \sigma_{n}^{B}$. In sum, $\partial B_{n}^{c}(\bar{\sigma}) / \partial \bar{\sigma}=0$ iff $\bar{\sigma} \leq \sigma_{n}^{B}$ and $\partial B_{n}^{c}(\bar{\sigma}) / \partial \bar{\sigma}<0$ iff $\bar{\sigma}>\sigma_{n}^{B}$.

(ii) (b) Proof.

As in point (ii) (a), if $\sigma_{s}^{B}>\bar{\sigma}$, then $\partial B_{s}^{c} / \partial \bar{\sigma}=0$. So let us focus on the case where the search requirement is binding. Following similar arguments as in point (ii) (a), we obtain:

$$
B_{s}^{c}(\bar{\sigma})=u(b)-c(\bar{\sigma})+\beta \delta\left\{\frac{\lambda(\bar{\sigma})}{1-\delta(1-q)} Q[r(\bar{\sigma} \mid b)]+\frac{u[r(\bar{\sigma} \mid b)]}{1-\delta}\right\}
$$

Partially differentiating $B_{s}^{c}(\bar{\sigma})$ in A.9 with respect to $\bar{\sigma}$ then yields

$$
\frac{\partial B_{s}^{c}(\bar{\sigma})}{\partial \bar{\sigma}}=S(\bar{\sigma}, r(\bar{\sigma} \mid b) \mid \beta)+\beta \delta \frac{[(1-\delta)(1-h[\bar{\sigma}, r(\bar{\sigma} \mid b)]+\delta q)] u^{\prime}\left[r\left(\sigma \mid y^{U}\right)\right]}{[1-\delta(1-q)](1-\delta)} \frac{\partial r(\bar{\sigma} \mid b)}{\partial \bar{\sigma}}
$$

First, consider $\bar{\sigma}=\sigma_{s}^{B}$. Then $\partial B_{s}^{c}(\bar{\sigma}) / \partial \bar{\sigma}>0$, since $S\left(\sigma_{s}^{B}, r\left(\sigma_{s}^{B} \mid b\right) \mid \beta\right)=0$ and, by Proposition 1 (ii), $\partial r(\bar{\sigma} \mid b) / \partial \bar{\sigma}>0$ iff $\bar{\sigma}<\sigma_{e}^{B}$. Now, consider $\bar{\sigma}=\sigma_{e}^{B}$. Then $\partial B_{s}^{c}(\bar{\sigma}) / \partial \bar{\sigma}<0$, since $S(\sigma, r(\sigma \mid b) \mid \beta)<0$ for all $\sigma>\sigma_{s}^{B}$ and, by Proposition 1 (ii), $\partial r(\bar{\sigma} \mid b) / \partial \bar{\sigma}=0$ iff $\bar{\sigma}=\sigma_{e}^{B}$. As $\partial B_{s}^{c}(\bar{\sigma}) / \partial \bar{\sigma}$ is a continuous function, the proposition must hold.

(iii)

We prove (iii) by showing that for any $\bar{\sigma} \geq \sigma_{j}^{B}$ the difference between the expected lifetime utility of a complying and a non-complying current self must always be strictly larger for an agent discounting the future at an exponential rate $(\beta=1)$ than for an agent discounting the future at a hyperbolic rate $(\beta<1)$. Consequently the hyperbolic agent will always stop complying (when $B_{j}^{c}\left(\bar{\sigma}_{j}^{\max }\right)=\tilde{Z}_{j}^{c}$ ) at a lower level of search effort than that of an exponential agent. Note that the expected lifetime utility for an agent discounting the future at an exponential rate is equal to the utility of the future self to which, in case of non-compliance, one adjusts for the fact that the current self is not yet sanctioned and earns therefore $b$ instead of $z$, i.e. is equal to $B_{e}^{f}(\bar{\sigma})$ if one complies and to $\tilde{Z}_{e}^{f} \equiv Z_{e}^{f}+u(b)-u(z)$ if one does not comply.

(a) Proof.

(1) Using (2), 9 and (10), and restricting the analysis to the cases where $\bar{\sigma} \geq \sigma_{n}^{B}$, we obtain:

$$
\forall \bar{\sigma}<\sigma_{e}^{B}: B_{n}^{f}(\bar{\sigma})-B_{n}^{c}(\bar{\sigma})=\delta(1-\beta)\left\{\frac{\lambda(\bar{\sigma})}{[1-\delta(1-q)]} Q\left(x_{e}^{B}\right)+\frac{u\left(x_{e}^{B}\right)}{(1-\delta)}\right\}
$$


and

$$
\forall \bar{\sigma} \geq \sigma_{e}^{B}: B_{n}^{f}(\bar{\sigma})-B_{n}^{c}(\bar{\sigma})=\delta(1-\beta)\left\{\frac{\lambda(\bar{\sigma})}{[1-\delta(1-q)]} Q(r(\bar{\sigma} \mid b))+\frac{u(r(\bar{\sigma} \mid b))}{(1-\delta)}\right\}
$$

Similarly, using (3), (9) and (10), we find

$$
\tilde{Z}_{n}^{f}-\tilde{Z}_{n}^{c}=\delta(1-\beta)\left\{\frac{\lambda\left(\sigma_{n}^{Z}\right)}{[1-\delta(1-q)]} Q\left(x_{e}^{Z}\right)+\frac{u\left(x_{e}^{Z}\right)}{(1-\delta)}\right\}
$$

(2) Consider definition (11) and assume that $x_{1}<x_{2}$. Then we can rewrite $Q\left(x_{1}\right)$ as follows:

$$
\begin{aligned}
Q\left(x_{1}\right) & =\int_{x_{1}}^{x_{2}}\left[u(w)-u\left(x_{1}\right)\right] d F(w)+Q\left(x_{2}\right)+\bar{F}\left(x_{2}\right)\left[u\left(x_{2}\right)-u\left(x_{1}\right)\right] \\
& =Q\left(x_{2}\right)+\bar{F}\left(x_{1}\right)\left[u\left(x_{2}\right)-u\left(x_{1}\right)\right]-\left\{\left[u\left(x_{2}\right)-u\left(x_{1}\right)\right]-E\left[u(w)-u\left(x_{1}\right) \mid x_{1} \leq w<x_{2}\right]\right\} \\
& \times\left[\bar{F}\left(x_{1}\right)-\bar{F}\left(x_{2}\right)\right]
\end{aligned}
$$

(3) Since $x_{e}^{B}>x_{e}^{Z}$, we can use 11 and A.13 to rewrite A.12 as follows:

$$
\begin{aligned}
\tilde{Z}_{n}^{f}-\tilde{Z}_{n}^{c} & =\frac{\delta(1-\beta)}{[1-\delta(1-q)]}\left\{\lambda\left(\sigma_{n}^{Z}\right) Q\left(x_{e}^{B}\right)+h\left(\sigma_{n}^{Z}, x_{e}^{B}\right)\left[u\left(x_{e}^{B}\right)-u\left(x_{e}^{Z}\right)\right]+u\left(x_{e}^{Z}\right)\left[1+\frac{\delta q}{1-\delta}\right]\right. \\
& \left.-\left[u\left(x_{e}^{B}\right)-u\left(x_{e}^{Z}\right)\right]-E\left[u(w)-u\left(x_{e}^{Z}\right) \mid x_{e}^{Z} \leq w<x_{e}^{B}\right]\left[\bar{F}\left(x_{e}^{Z}\right)-\bar{F}\left(x_{e}^{B}\right)\right]\right\} \quad \text { (A.14) }
\end{aligned}
$$

Subtracting A.14 from A.10 then yields for $\bar{\sigma} \in\left[\sigma_{n}^{Z}, \sigma_{e}^{B}\right]$ :

$$
\begin{aligned}
& B_{n}^{f}(\bar{\sigma})-B_{n}^{c}(\bar{\sigma})-\tilde{Z}_{n}^{f}+\tilde{Z}_{n}^{c}=\frac{\delta(1-\beta)}{[1-\delta(1-q)]}\left\{Q\left(x_{e}^{B}\right)\left[\lambda(\bar{\sigma})-\lambda\left(\sigma_{n}^{Z}\right)\right]+\left[u\left(x_{e}^{B}\right)-u\left(x_{e}^{Z}\right)\right]\right. \\
& \times \frac{\left[(1-\delta)\left(1-h\left(\sigma_{n}^{Z}, x_{e}^{B}\right)\right)+\delta q\right]}{(1-\delta)}+\left\{\left[u\left(x_{e}^{B}\right)-u\left(x_{e}^{Z}\right)\right]-E\left[u(w)-u\left(x_{e}^{Z}\right) \mid x_{e}^{Z} \leq w<x_{e}^{B}\right]\right\} \\
& \left.\times\left[\bar{F}\left(x_{e}^{Z}\right)-\bar{F}\left(x_{e}^{B}\right)\right]\right\}>0
\end{aligned}
$$

Since $\forall \bar{\sigma} \in\left[\sigma_{e}^{B}, \bar{\sigma}_{n(e)}^{\max }\right]: r(\bar{\sigma} \mid b)>x_{e}^{Z}$, we can derive using A.11 a similar expression as A.15 in which $x_{e}^{B}$ is replaced by $r(\bar{\sigma} \mid b)$. Consequently,

$$
\forall \bar{\sigma} \in\left[\sigma_{n}^{Z}, \bar{\sigma}_{n(e)}^{\max }\right]: B_{n}^{f}(\bar{\sigma})-\tilde{Z}_{n}^{f}>B_{n}^{c}(\bar{\sigma})-\tilde{Z}_{n}^{c}
$$

Since $B_{n}^{f}\left(\bar{\sigma}_{n(e)}^{\max }\right)=\tilde{Z}_{n}^{f}$, A.16 implies that $B_{n}^{c}\left(\bar{\sigma}_{n(e)}^{\max }\right)<\tilde{Z}_{n}^{c}$. Because $B_{n}^{c}\left(\sigma_{n}^{Z}\right)>\tilde{Z}_{n}^{c}$ and $B_{n}^{c}(\cdot)$ is a continuous function, it must be that $\sigma_{n}^{Z}<\bar{\sigma}_{n}^{\max }<\bar{\sigma}_{n(e)}^{\max }$.

(b) Proof.

(1) Using (2), (9) and (10), and restricting the analysis to the cases where $\bar{\sigma} \geq \sigma_{s}^{B}$, we obtain

$$
\forall \bar{\sigma}: B_{s}^{f}(\bar{\sigma})-B_{s}^{c}(\bar{\sigma})=\delta(1-\beta)\left\{\frac{\lambda(\bar{\sigma})}{[1-\delta(1-q)]} Q(r(\bar{\sigma} \mid b))+\frac{u(r(\bar{\sigma} \mid b))}{(1-\delta)}\right\}
$$

Following a similar reasoning, we find

$$
Z_{s}^{f}(\bar{\sigma})-Z_{s}^{c}(\bar{\sigma})=\delta(1-\beta)\left\{\frac{\lambda\left(\sigma_{s}^{Z}\right)}{[1-\delta(1-q)]} Q\left(x_{s}^{Z}\right)+\frac{Q\left(x_{s}^{Z}\right)}{(1-\delta)}\right\}
$$


(2) Since $\forall \bar{\sigma} \in\left[\sigma_{s}^{Z}, \bar{\sigma}_{s(e)}^{\max }\right]: r(\bar{\sigma} \mid b)>x_{s}^{Z}$, we can derive, using A.13 and a similar derivation as in part (a)(3), an expression like A.15 for $\bar{\sigma} \in\left[\sigma_{s}^{Z}, \bar{\sigma}_{s(e)}^{\max }\right]$ :

$$
\begin{aligned}
& B_{s}^{f}(\bar{\sigma})-B_{s}^{c}(\bar{\sigma})-\tilde{Z}_{s}^{f}+\tilde{Z}_{s}^{c}=\frac{\delta(1-\beta)}{[1-\delta(1-q)]}\left\{Q[r(\bar{\sigma} \mid b)]\left[\lambda(\bar{\sigma})-\lambda\left(\sigma_{s}^{Z}\right)\right]\right. \\
& +\left[u[r(\bar{\sigma} \mid b)]-u\left(x_{s}^{Z}\right)\right] \times \frac{\left[(1-\delta)\left(1-h\left(\sigma_{s}^{Z}, r(\bar{\sigma} \mid b)\right)\right)+\delta q\right]}{(1-\delta)} \\
& \left.+\left\{\left[u[r(\bar{\sigma} \mid b)]-u\left(x_{s}^{Z}\right)\right]-E\left[u(w)-u\left(x_{s}^{Z}\right) \mid x_{s}^{Z} \leq w<r(\bar{\sigma} \mid b)\right]\right\}\left[\bar{F}\left(x_{s}^{Z}\right)-\bar{F}[r(\bar{\sigma} \mid b)]\right]\right\}>0
\end{aligned}
$$

Therefore,

$$
\forall \bar{\sigma} \in\left[\sigma_{s}^{Z}, \bar{\sigma}_{s(e)}^{\max }\right]: B_{s}^{f}(\bar{\sigma})-\tilde{Z}_{s}^{f}>B_{s}^{c}(\bar{\sigma})-\tilde{Z}_{s}^{c}
$$

Since $B_{s}^{f}\left(\bar{\sigma}_{s(e)}^{\max }\right)=\tilde{Z}_{s}^{f}$, A.19 implies that $B_{s}^{c}\left(\bar{\sigma}_{s(e)}^{\max }\right)<\tilde{Z}_{s}^{c}$. Because $B_{s}^{c}\left(\sigma_{s}^{Z}\right)>\tilde{Z}_{s}^{c}$ and $B_{s}^{c}(\cdot)$ is a continuous function, it must be that $\sigma_{s}^{Z}<\bar{\sigma}_{s}^{\max }<\bar{\sigma}_{s(e)}^{\max }$.

(3) Let $\tilde{\sigma}_{s}$ solve $B_{s}^{c}\left(\tilde{\sigma}_{s}\right)=B_{s}^{c}$. As $B_{s}^{c}>\tilde{Z}_{s}^{c}$, it follows that $B_{s}^{c}\left(\tilde{\sigma}^{s}\right)>\tilde{Z}_{s}^{c}$. Therefore, $\bar{\sigma}_{s}^{\max }>\max \left\{\sigma_{s}^{Z}, \tilde{\sigma}_{s}\right\}$.

\section{Proof of Proposition 3}

We prove uniqueness for the solution of the complying agent. Uniqueness for the non-complying agent follows immediately by replacing $B$ by $Z$.

(i) The Sophisticated Agent

Proof.

(a) The Interior Solution

The interior solution for the sophisticated agent is found at the intersection of $r(\sigma \mid b)$ and $s(\sigma \mid \beta<1)$. Since, by Proposition 1 (i) and (ii), $r(\sigma \mid b)$ must be strictly increasing and $s(\sigma \mid \beta<1)$ strictly decreasing in $\sigma$ for any $\sigma<\sigma_{e}^{B}$. This means that there can be only one intersection for any $\sigma<\sigma_{e}^{B}$.

Imagine that $r(\sigma \mid b)$ crosses $s(\sigma \mid \beta<1)$ for $\sigma>\sigma_{e}^{B}$, i.e. for values such that $r(\sigma \mid b)$ is strictly declining in $\sigma$. Then, $r(\sigma \mid b)$ has necessarily also an intersection with $s(\sigma \mid \beta=1)$ in the same region where $\sigma>\sigma_{e}^{B}$. However, this is impossible since at this crossing point $r(\sigma \mid b)$ cannot be strictly decreasing (its derivative being at this point $S(\sigma, x \mid 1)=0$ ).

(b) The Constrained Solution

The constrained solution for the sophisticated agent is found at the intersection of $r(\sigma \mid b)$ and the vertical $\sigma=\bar{\sigma}$. Since, by Proposition 1 (ii), $r(\sigma \mid b)$ is never vertical, the solution is unique. 
(ii) The Naive Agent

Proof.

(a) The Interior Solution

The interior solution for the naive agent is found in two steps. One first solves for the exponential agent and then takes the reservation wage of the exponential agent as given to solve for the optimal search effort. The solution of the exponential agent is found at the intersection of $r(\sigma \mid b)$ and $s(\sigma \mid 1)$. Since, by Proposition 1 (i) and (ii), $s(\sigma \mid 1$ ) is strictly decreasing and $r(\sigma \mid b)$ must be horizontal at the point of intersection with $s(\sigma \mid 1)$, there can be only one intersection. Second, the solution of the naive agent is found at the intersection between $s(\sigma \mid \beta<1)$, which is strictly decreasing, and $x_{n}^{B}=x_{e}^{B}$, which is horizontal in the $(\sigma, x)$-plane. The solution must therefore be unique.

(b) The Constrained Solution

The constrained solution for the sophisticated agent is found at the intersection between the vertical $\sigma=\bar{\sigma}$ and the horizontal $x_{n}^{B}=x_{e}^{B}$ if $\sigma \leq \sigma_{e}^{B}$ or the strictly decreasing curve $r(\sigma \mid b)$ for $\sigma>\sigma_{e}^{B}$ (see Proposition 1 (ii)). The solution must therefore be unique.

\section{E Proof of Proposition 5}

Below we show that, for the sophisticated agent, imposing a minimum search effort $\bar{\sigma}>\sigma_{s}^{B}$ to be eligible to UI has a positive effect on the exit rate out of unemployment. This amounts to sign the following expression:

$$
\frac{d h[\bar{\sigma}, r(\bar{\sigma} \mid b)]}{d \bar{\sigma}}=(1-F[r(\bar{\sigma} \mid b)]) \lambda^{\prime}(\bar{\sigma})-\lambda(\bar{\sigma}) f[r(\bar{\sigma} \mid b)] \frac{\left[-c^{\prime}(\bar{\sigma})+\frac{\lambda^{\prime}(\bar{\sigma}) \delta}{1-\delta(1-q)} Q[r(\bar{\sigma} \mid b)]\right]}{u^{\prime}[r(\bar{\sigma} \mid b)]\left[1+\frac{\lambda(\bar{\sigma}) \delta}{1-\delta(1-q)}(1-F[r(\bar{\sigma} \mid b)])\right]}
$$

Proof. This proof concerns individuals who are complying to the job requirement. To avoid clutter, in the sequel we will write $x$ for $r(\bar{\sigma} \mid b)$ and drop the superscript $B$. For $\bar{\sigma}>\sigma_{e}, d h / d \sigma>0$ because $d x / d \bar{\sigma}<0$ for $\bar{\sigma}>\sigma_{e}^{B}$. For $\bar{\sigma}=\sigma_{e}$, the expression A.20 becomes:

$$
\frac{d h}{d \bar{\sigma}}\left(\sigma_{e}, x_{e}\right)=\left[1-F\left(x_{e}\right)\right] \lambda^{\prime}\left(\sigma_{e}\right)>0
$$

because $d x /\left.d \bar{\sigma}\right|_{R=0}=0$ at $\bar{\sigma}=\sigma_{e}$. It remains to be shown that $\partial h / \partial \bar{\sigma}>0$ also for $\bar{\sigma} \in\left[\sigma_{s}, \sigma_{e}\right)$. To do this, we will proceed in three steps:

(i) we provide sufficient conditions such that $\partial h / \partial \bar{\sigma}>0$ at $\bar{\sigma}=\sigma_{s}$; 
(ii) we will show that $h\left(\sigma_{e}, x_{e}\right)>h\left(\sigma_{s}, x_{s}\right)$, by extending Proposition 3 of PDV to risk aversion: ${ }^{18}$

(iii) we will extend result (i) to all pairs $(\bar{\sigma}, x)$ satisfying $R(\cdot, \cdot \mid b)=0$ when $\bar{\sigma} \in\left(\sigma_{s}, \sigma_{e}\right)$.

(i) To show that $d h\left(\sigma_{s}, x_{s}\right) / d \bar{\sigma}=(1-F(x)) \lambda^{\prime}(\bar{\sigma})-\lambda(\bar{\sigma}) f(x) \cdot d x /\left.d \bar{\sigma}\right|_{R=0}>0$ we follow a similar approach to the one used by van den Berg (1994, p.493). To simplify the notation we define the parameter $1 / \rho=\delta /[1-\delta(1-q)]$. Using the F.O.C. of search for the sophisticated agent, the slope of curve $r(\bar{\sigma} \mid b)$ at $\left(\sigma_{s}, x_{s}\right)$ can be written as:

$$
\frac{d x}{d \bar{\sigma}}\left(\sigma_{s}, x_{s}\right)=\frac{\lambda^{\prime}\left(\sigma_{s}\right)(1-\beta) Q\left(x_{s}\right)}{u^{\prime}\left(x_{s}\right)\left(\rho+h\left(\sigma_{s}, x_{s}\right)\right)}
$$

Denoting $\psi\left(x_{s}\right)=f\left(x_{s}\right) / \bar{F}\left(x_{s}\right)$ the hazard rate,

$$
\begin{aligned}
\frac{d h}{d \bar{\sigma}}\left(\sigma_{s}, x_{s}\right) & =\bar{F}\left(x_{s}\right) \lambda^{\prime}\left(\sigma_{s}\right)-\lambda\left(\sigma_{s}\right) f\left(x_{s}\right)\left[\frac{\lambda^{\prime}\left(\sigma_{s}\right)(1-\beta) Q\left(x_{s}\right)}{u^{\prime}\left(x_{s}\right)\left(\rho+h\left(\sigma_{s}, x_{s}\right)\right)}\right] \\
& =\underbrace{\frac{\lambda^{\prime}\left(\sigma_{s}\right)}{u^{\prime}\left(x_{s}\right)\left[\rho+h\left(\sigma_{s}, x_{s}\right)\right]}}_{>0}\left\{\bar{F}\left(x_{s}\right) u^{\prime}\left(x_{s}\right)\left[\rho+h\left(\sigma_{s}, x_{s}\right)\right]-h\left(\sigma_{s}, x_{s}\right) \psi\left(x_{s}\right)(1-\beta) Q\left(x_{s}\right)\right\}(\mathrm{A}
\end{aligned}
$$

So,

$$
\frac{d h}{d \bar{\sigma}}\left(x_{s}, \sigma_{s}\right) \geq 0 \Leftrightarrow \frac{\rho}{h\left(\sigma_{s}, x_{s}\right)}+1 \geq \frac{\psi\left(x_{s}\right)(1-\beta) Q\left(x_{s}\right)}{u^{\prime}\left(x_{s}\right) \bar{F}(x)}
$$

Define the conditional expectation $\mu(x)=E_{F}(u(w) \mid w>x)=\left[\int_{x}^{\bar{w}} u(w) f(w) d w\right] / \bar{F}(x)$. Equation (14) can be rewritten as $\rho / h\left(\sigma_{s}, x_{s}\right)=\left[\mu\left(x_{s}\right)-u\left(x_{s}\right)\right] /\left[u\left(x_{s}\right)-\left(u(b)-c\left(\sigma_{s}\right)\right)\right]$. Plugging the latter into A.23, $d h\left(\sigma_{s}, x_{s}\right) / d \bar{\sigma}>0$ if

$$
\frac{\mu\left(x_{s}\right)-\left[u(b)-c\left(\sigma_{s}\right)\right]}{u\left(x_{s}\right)-\left[u(b)-c\left(\sigma_{s}\right)\right]} \geq \frac{\psi\left(x_{s}\right)(1-\beta) Q\left(x_{s}\right)}{u^{\prime}\left(x_{s}\right) \bar{F}(x)}
$$

Now, the left-hand side of A.24 is bigger than $\mu\left(x_{s}\right) / u\left(x_{s}\right)$ if $u\left(x_{s}\right)-\left[u(b)-c\left(\sigma_{s}\right)\right]>0$, which is true at the optimum by (14), and if $\left[u(b)-c\left(\sigma_{s}\right)\right]>0$. The latter condition, which implies that the utility level is positive, is the first assumption of Proposition 5. Since $\beta \leq 1$, a sufficient condition for A.24 is

$$
\frac{\mu\left(x_{s}\right)}{u\left(x_{s}\right)} \geq \frac{\psi\left(x_{s}\right) Q\left(x_{s}\right)}{u^{\prime}\left(x_{s}\right) \bar{F}(x)} \Leftrightarrow \mu\left(x_{s}\right) \bar{F}(x) \geq \frac{u\left(x_{s}\right) \psi\left(x_{s}\right) Q\left(x_{s}\right)}{u^{\prime}\left(x_{s}\right)}
$$

Notice that $\mu\left(x_{s}\right) \bar{F}\left(x_{s}\right)=\int_{x_{s}}^{\bar{w}} u(w) f(w) d w=\int_{x_{s}}^{\bar{w}} u(w) \psi(w) \bar{F}(w) d w$, whereas $Q\left(x_{s}\right)=\int_{x_{s}}^{\bar{w}} \bar{F}(w) u^{\prime}(w) d w$ by integration by parts. Expression (A.25) can then be rewritten as:

$$
\int_{x_{s}}^{\bar{w}} u(w) \psi(w) \bar{F}(w) d w \geq \frac{u\left(x_{s}\right) \psi\left(x_{s}\right)}{u^{\prime}\left(x_{s}\right)} \int_{x_{s}}^{\bar{w}} \bar{F}(w) u^{\prime}(w) d w
$$

\footnotetext{
${ }^{18}$ Proposition 3 of PDV states that under risk neutrality, the exit rate at the optimum of a sophisticated worker is increasing in $\beta$ if $d\left[E_{F}(w \mid w>x)\right] / d x \leq 1 /(1-\beta)$ at $x=x_{s}$. According to PDV, for $\beta=2 / 3$, which is in the lower range of estimates for $\beta$, this condition requires that $d\left[E_{F}(w \mid w>x)\right] / d x \leq 3$ at $x=x_{s}$, which is always satisfied in the class of log-concave wage distributions.
} 
or

$$
\int_{x_{s}}^{\bar{w}} u^{\prime}(w)\left[\frac{u(w)}{u^{\prime}(w)} \psi(w)-\frac{u\left(x_{s}\right)}{u^{\prime}\left(x_{s}\right)} \psi\left(x_{s}\right)\right] \bar{F}(w) d(w) \geq 0
$$

This condition is satisfied if $\left[u(w) / u^{\prime}(w)\right] \psi(w)$ increases on $(x, \bar{w})$. Equivalently, $\log [u(w)]-\log \left[u^{\prime}(w)\right]+$ $\log [\psi(w)]$ has to increase on the same interval, i.e.

$$
\frac{d \log \psi(w)}{d \log w} \geq-\frac{d \log u(w)}{d \log w}+\frac{d \log u^{\prime}(w)}{d \log w}
$$

on $\left(x_{s}, \bar{w}\right)$. This condition (assumption 2 of Proposition 5) is further discussed in the core of the paper.

(ii) To show that $h\left(\sigma_{e}, x_{e}\right)>h\left(\sigma_{s}, x_{s},\right)$, we prove $d h\left(\sigma_{s}, x_{s}\right) / d \beta>0$ for $u(\cdot)$ concave. Define

$$
\Delta=u^{\prime}\left(x_{s}\right)\left(\frac{\lambda\left(\sigma_{s}\right) \bar{F}\left(x_{s}\right)}{\rho}+1\right)\left(\frac{\beta \lambda^{\prime \prime}\left(\sigma_{s}\right) Q\left(x_{s}\right)}{\rho}-c^{\prime \prime}\left(\sigma_{s}\right)\right)-\frac{(1-\beta) \beta \lambda^{\prime}\left(\sigma_{s}\right) Q\left(x_{s}\right) u^{\prime}\left(x_{s}\right) \lambda\left(\sigma_{s}\right)}{\rho}<0
$$

Totally differentiating the system of (14) and (15) gives:

$$
\begin{gathered}
\frac{d \sigma_{s}}{d \beta}=-\frac{1}{\Delta}\left(\frac{\lambda^{\prime}\left(\sigma_{s}\right) Q\left(x_{s}\right) u^{\prime}\left(x_{s}\right)}{\rho}\right)\left(\frac{\lambda\left(\sigma_{s}\right) \bar{F}\left(x_{s}\right)}{\rho}+1\right)>0 \\
\frac{d x_{s}}{d \beta}=-\frac{1}{\Delta}(1-\beta)\left(\frac{\lambda^{\prime}\left(\sigma_{s}\right) Q\left(x_{s}\right)}{\rho}\right)^{2} \geq 0
\end{gathered}
$$

Plugging A.28 and A.29 into $d h\left(\sigma_{s}, x_{s}\right) / d \beta$, the latter becomes:

$$
\frac{d h\left(\sigma_{s}, x_{s}\right)}{d \beta}=\left(\frac{\left[\lambda^{\prime}\left(\sigma_{s}\right)\right]^{2} Q\left(x_{s}\right)}{\Delta \rho^{2}}\right)\left\{(1-\beta) f\left(x_{s}\right) Q\left(x_{s}\right) \lambda\left(\sigma_{s}\right)-\bar{F}\left(x_{s}\right) u^{\prime}\left(x_{s}\right)\left[\lambda\left(\sigma_{s}\right) \bar{F}\left(x_{s}\right)+\rho\right]\right\}
$$

Since $\Delta<0$, a sufficient condition for this expression to be positive is that

$$
\frac{f\left(x_{s}\right)}{\bar{F}\left(x_{s}\right)}(1-\beta) Q\left(x_{s}\right) \lambda\left(\sigma_{s}\right) \leq u^{\prime}\left(x_{s}\right)\left[\lambda\left(\sigma_{s}\right) \bar{F}\left(x_{s}\right)+\rho\right]
$$

Dividing and multiplying the L.H.S. by $\bar{F}\left(x_{s}\right)$, expression A.30 can be written as:

$$
\psi\left(x_{s}\right)(1-\beta) \frac{Q\left(x_{s}\right)}{\bar{F}\left(x_{s}\right)} h\left(x_{s}, \sigma_{s}\right) \leq u^{\prime}\left(x_{s}\right)\left[\lambda\left(\sigma_{s}\right) \bar{F}\left(x_{s}\right)+\rho\right]
$$

Notice that condition A.31 is exactly condition A.23 of part (i), which is satisfied under assumption 2 of the proposition. Thus, we can conclude that $d h\left(\sigma_{s}, x_{s}\right) / d \beta \geq 0$ under this assumption.

(iii) We now extend result (i) to all pairs $(\bar{\sigma}, x)$ satisfying $R(\cdot, \cdot \mid b)=0$ with $\bar{\sigma} \in\left(\sigma_{s}, \sigma_{e}\right)$. Those constrained choices can be reinterpreted as the unconstrained optimum of a sophisticated agent whose short-term discount factor is higher than $\beta$. Hence, result (i) can be applied so that the proposition also holds for $\bar{\sigma} \in\left(\sigma_{s}, \sigma_{e}\right)$. 


\section{F Proof of Proposition 6}

(i) Proof.

The proposition follows directly from partially differentiating (28) with respect to $\sigma$ and from the fact that $\forall \sigma \lesseqgtr \sigma_{e}^{P}(\bar{\sigma}): S_{p}\left[\sigma, r_{p}(\sigma \mid b, \bar{\sigma}) \mid 1, \bar{\sigma}\right] \gtreqless 0$.

(ii) Proof.

Partially differentiating (28) with respect to $\sigma$ yields:

$$
\frac{\partial s_{p}(\sigma \mid \beta, \bar{\sigma})}{\partial \sigma}=\frac{\left\{\beta \delta\left[\frac{\lambda^{\prime \prime}(\sigma) Q\left[s_{p}(\sigma \mid \beta, \bar{\sigma})\right]}{1-\delta(1-q)}-\frac{\partial^{2} p\left(\frac{\bar{\sigma}}{\sigma}\right)}{[\partial \sigma]^{2}}\left[u\left[s_{p}(\sigma \mid \beta, \bar{\sigma})\right]-u\left(x_{j}^{Z}\right)\right]\right]-c^{\prime \prime}(\sigma)\right\}[1-\delta(1-q)](1-\delta)}{\left[\lambda^{\prime}(\sigma) \bar{F}\left[s_{p}(\sigma \mid \beta, \bar{\sigma})\right](1-\delta)+\frac{\partial p\left(\frac{\bar{\sigma}}{\sigma}\right)}{\partial \sigma}[1-\delta(1-q)]\right] \beta \delta u^{\prime}\left[s_{p}(\sigma \mid \beta, \bar{\sigma})\right]}
$$

If $\partial^{2}[p(\bar{\sigma} / \sigma)] /[\partial \sigma]^{2} \geq 0$ then the numerator of $\mathrm{A.32}$ is strictly negative, so that the sign of $\partial s_{p}(\sigma \mid \beta, \bar{\sigma}) / \partial \sigma$ is equal to the sign of $-[\partial p(\bar{\sigma} / \sigma) / \partial \sigma][1-\delta(1-q)]-\lambda^{\prime}(\sigma) \bar{F}\left[s_{p}(\sigma \mid \beta, \bar{\sigma})\right](1-$ $\delta)$. If $\partial s_{p}(\sigma \mid \beta, \bar{\sigma}) / \partial \sigma<0$, then the slope of $s_{p}(\sigma \mid \beta, \bar{\sigma})$ is more negative than that of $s(\sigma \mid \beta)$, since the numerator is more negative and the denominator is smaller.

(iii) and (iv)

Proof.

This is found by directly partially differentiating (27) and 28) with respect to $b, \beta$ and $\bar{\sigma}$. 